\title{
Optically active imidazole N-oxides derived from L-prolinamine
}

\author{
Mlostoń, Grzegorz ; Wroblewska, Aneta ; Obijalska, Emilia ; Heimgartner, Heinz
}

\begin{abstract}
Starting with (S)-1-benzylprolinamine and alpha-hydroxyimino ketones, enantiomerically pure bisheterocyclic imidazole N-oxides bearing the (S)-configured N-benzyl(pyrrolidin-2-yl)methyl residue were prepared. These N-oxides reacted with 2,2,4,4-tetramethylcyclobutane-1,3-dithione to give the corresponding optically active imidazole-2-thione derivatives via a sulfur transfer reaction. Reduction of the N-oxides with Raney-nickel led to deoxygenation, whereas catalytic hydrogenation $(\mathrm{Pd} / \mathrm{C})$ in ethanol occurred with simultaneous deoxygenation and debenzylation, leading to optically active 1-(pyrrolidin-2yl)methyl-1H-imidazoles. Alkylation of the prepared imidazole N-oxides and their respective imidazoles with butyl and hexyl bromide and subsequent anion exchange gave optically active N-alkoxy- and N-alkylimidazolium tetrafluoroborates, respectively, with the properties of 'room temperature ionic liquids'.
\end{abstract}

DOI: https://doi.org/10.1016/j.tetasy.2013.07.013

Posted at the Zurich Open Repository and Archive, University of Zurich

ZORA URL: https://doi.org/10.5167/uzh-80783

Journal Article

Accepted Version

Originally published at:

Mlostoń, Grzegorz; Wroblewska, Aneta; Obijalska, Emilia; Heimgartner, Heinz (2013). Optically active imidazole N-oxides derived from L-prolinamine. Tetrahedron: Asymmetry, 24(15-16):958-965.

DOI: https://doi.org/10.1016/j.tetasy.2013.07.013 


\title{
Optically Active Imidazole $N$-Oxides Derived from L-Prolinamine ${ }^{\ddagger}$
}

\author{
Grzegorz MlostoD*, Aneta Wróblewska ${ }^{a^{*}}$, Emilia Obijalska ${ }^{\text {a, }}$ \\ and Heinz Heimgartner ${ }^{b^{*}}$ \\ ${ }^{a}$ University of Aódz, Department of Organic and Applied Chemistry, Tamka 12, \\ PL-91-403 Aódz, Poland \\ E-mail: gmloston@uni.lodz.pl \\ ${ }^{\mathrm{b}}$ Institute of Organic Chemistry, University of Zürich, \\ Winterthurerstrasse 190, CH-8057 Zürich, Switzerland
}

\begin{abstract}
Starting with (S)-1-benzylprolinamine and $\alpha$-hydroxyimino ketones, enantiomerically pure bisheterocyclic imidazole $N$-oxides bearing the $(S)$-configured $N$ benzyl(pyrrolidin-2-yl)methyl residue were prepared. These $N$-oxides reacted with 2,2,4,4-tetramethylcyclobutane-1,3-dithione to give the corresponding optically active imidazole-2-thione derivatives via sulfur transfer reaction. Reduction of the $\mathrm{N}$-oxides with Raney-nickel led to deoxygenation, whereas catalytic hydrogenation $(\mathrm{Pd} / \mathrm{C})$ in ethanol occurred with simultaneous deoxygenation and debenzylation, leading to optically active 1-(pyrrolidin-2-ylmethyl)-1H-imidazoles. Alkylation of the prepared imidazole $\mathrm{N}$-oxides and the respective imidazoles with butyl and hexylbromide and subsequent anion exchange gave optically active $\mathrm{N}$-alkoxy- and $\mathrm{N}$-alkylimidazolium tetrafluoroborates, respectively, with the properties of 'room temperature ionic liquids'.
\end{abstract}




\section{Introduction}

The importance of imidazole derivatives in organic, bioorganic, and medicinal chemistry as well as in materials science is well documented. ${ }^{2-4}$ In recent years, numerous reports on the synthesis and diverse applications of imidazole $N$-oxides were published. ${ }^{5}$ In contrast to six-membered N-heterocycles, imidazoles cannot be converted to the corresponding $\mathrm{N}$-oxides by treatment with typical oxidizing reagents. ${ }^{6}$ Therefore, condensations of $\sigma$-hydroxyimino ketones with methylidene amines (formaldehyde imines) were applied as a convenient access to 2 -unsubstituted imidazole $N$-oxides. ${ }^{5 a, 7}$ Starting with optically active primary amines, such as 1-phenylethylamine, transcyclohexane-1,2-diamine, as well as $\sigma$-amino acid esters, the desired enantiomerically pure imidazole N-oxides were obtained as sole products. ${ }^{8}$ Some of them were tested as ligands for asymmetric allylation of aldehydes ${ }^{9}$ and in cyclopropanation reactions. ${ }^{10}$

L-Prolinamine ((2S)-pyrrolidin-2-yl)methylamine) is a very useful and widely applied building block for the synthesis of optically active ligands of potential importance for asymmetric synthesis. ${ }^{11}$ The non-protected prolinamine easily reacts with electron-deficient dicyanofumarates, and enantiomerically pure bicyclic pyrazinones were obtained chemoselectively. ${ }^{12}$

The aim of the present study was the synthesis of new series of optically active imidazole derivatives containing the chiral pyrrolidine fragment originating from Lprolinamine. Some of the products were used for the preparation of new imidazolebased optically active ionic liquids.

\section{Results and discussion}

The preparation of the starting $N$-benzylated L-prolinamine (1) can be achieved from methyl L-prolinate via aminolysis and reduction of the amide ${ }^{13}$ or from L-prolinol via the intermediate azide. ${ }^{11 \mathrm{a}}$ In addition, we developed a new approach to $\mathbf{1 b}$ based on a Mitsunobu reaction via the intermediate phthalimide derivative (Scheme 1).

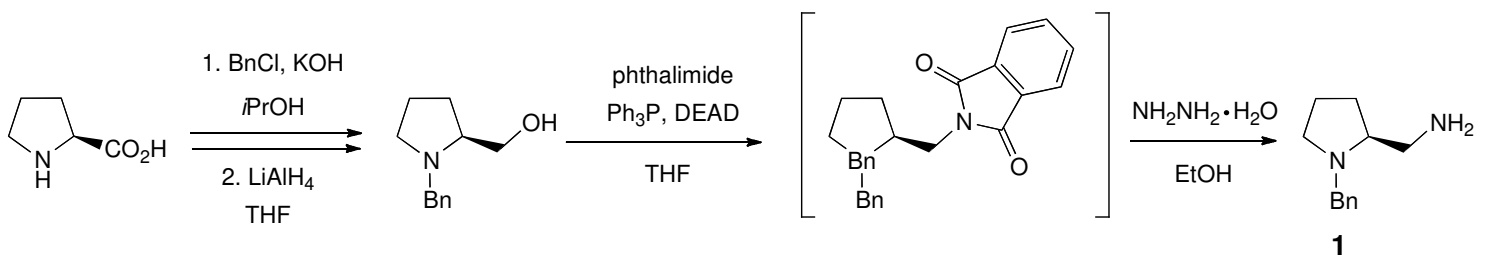

Scheme 1. 
The condensation of $\mathbf{1}$ with paraformaldehyde led to methylidene prolinamine $\mathbf{2}$, which spontaneously underwent trimerization to give the corresponding hexahydrotriazine 3 (Scheme 2). The structure of $\mathbf{3}$ was confirmed by the ${ }^{13} \mathrm{C}-\mathrm{NMR}$ spectrum, in which no signal for the imino group $\left(\mathrm{N}=\mathrm{CH}_{2}\right)$ appears. The trimerization of methylidene amines is a typical reaction, ${ }^{14}$ and only in the case of bulky substituents, e.g. adamantan-1-yl, the monomeric form is stable under standard conditions. ${ }^{15}$ In the case of the present study, the trimer $\mathbf{3}$ is believed to exist in solution in an equilibrium with the monomeric form 2.

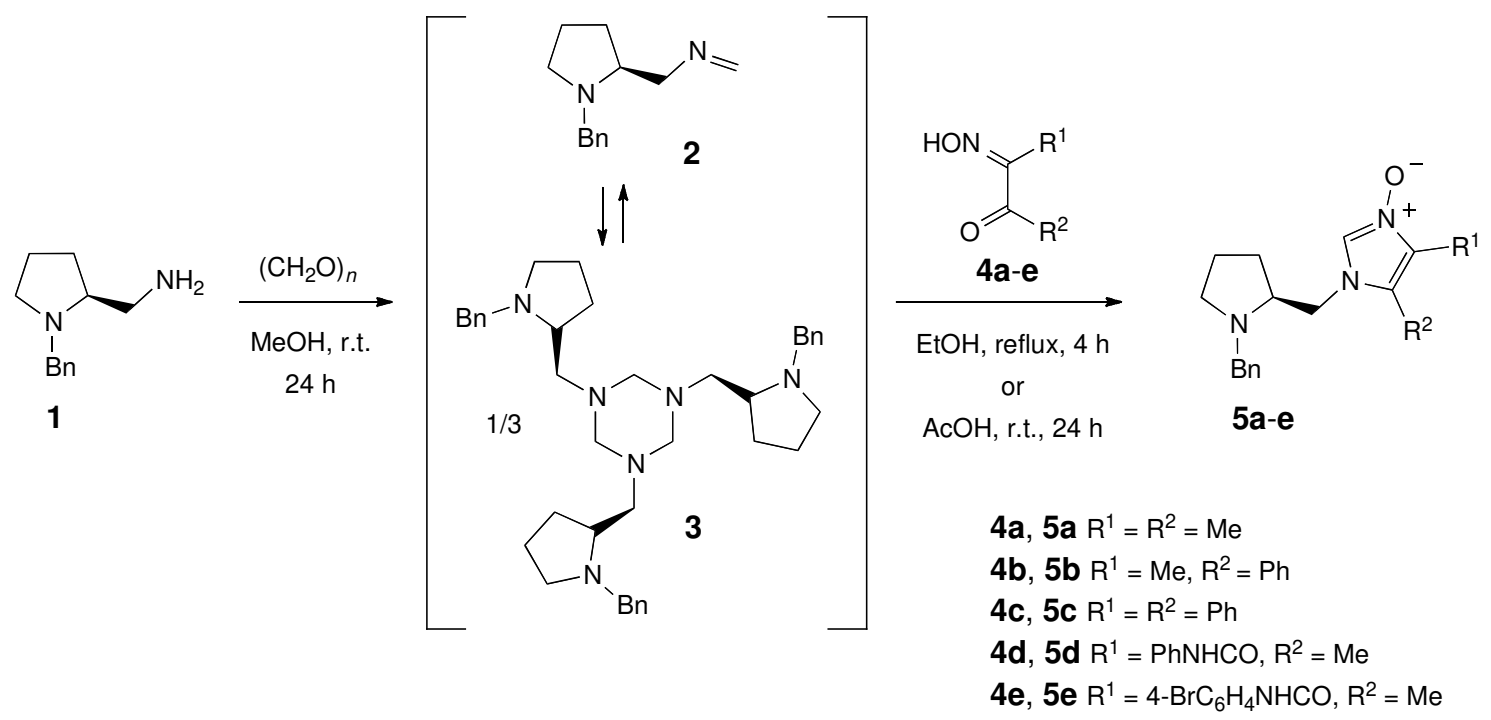

Scheme 2.

The latter reacts with $\sigma$-hydroxyimino ketones 4 a-e in boiling ethanol (method A) or in glacial acetic acid at room temperature (method B) yielding the desired optically active imidazole $N$-oxides $\mathbf{5 a - e}$ in good yields (Scheme 2). In order to examine the enantiopurity of these products, a sample of $\mathbf{5 a}$ was dissolved in $\mathrm{CDCl}_{3}$ with an equimolar amount of (tert-butyl)(phenyl)thiophosphinic acid (MOD reagent). ${ }^{16}$ The ${ }^{1} \mathrm{H}$ NMR spectrum showed only one set of signals, whereas in the case of racemic 5a (rac5a), addition of MOD led to the appearance of two sets. Thus, in the racemic product, the diagnostic $\mathrm{H}-\mathrm{C}(2)$ signal of imidazole $N$-oxides appeared as two singlets located at 8.87 and $8.86 \mathrm{ppm}$. Similarly, one Me group gave two singlets at 1.95 and $1.94 \mathrm{ppm}$, whereas the second Me group of both enantiomers absorbed as a singlet at $2.08 \mathrm{ppm}$. Moreover, for all other signals observed, duplication was observed in the spectrum of rac-5a. 
Based on this result, we assumed that all prepared imidazole $N$-oxides 5 are enantiomerically pure. The same conclusion was formulated in the case of other optically active imidazole $N$-oxides described in our previous papers. ${ }^{8}$

2-Unsubstituted imidazole $\mathrm{N}$-oxides are versatile starting materials for the preparation of other imidazole derivatives, such as the parent imidazoles, ${ }^{8}$ imidazole-2thiones, ${ }^{8}$ imidazolones, ${ }^{17}$ and (imidazol-2-yl)acetates. ${ }^{18}$ In addition, imidazole $N$-oxides or the corresponding parent compounds have been alkylated to give imidazolium salts, which display properties of ionic liquids. ${ }^{8 \mathrm{~d}, 19}$

Treatment of imidazole $N$-oxides 5 with Raney-Ni in ethanol at room temperature led smoothly to selective deoxygenation to yield the corresponding imidazoles 6 (Scheme 3). Under these conditions, no debenzylation of the pyrrolidine ring was observed. In a control experiment, the reaction mixture was heated to reflux, and in this case, a complex mixture of products was formed. On the other hand, hydrogenolysis $\left(\mathrm{H}_{2}, \mathrm{Pd} / \mathrm{C}\right)$ of $\mathbf{5 c}$ and $\mathbf{5 d}$ in ethanol led to deoxygenation and debenzylation to yield 7a and $\mathbf{7 b}$, respectively.

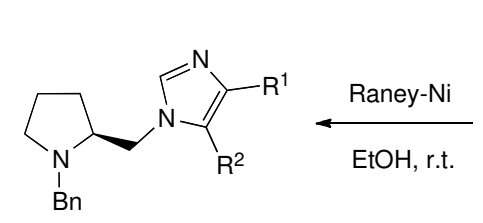

6a-e

6a $R^{1}=R^{2}=M e$

$6 b R^{1}=M e, R^{2}=P h$

6c $R^{1}=R^{2}=P h$

6d $\mathrm{R}^{1}=\mathrm{PhNHCO}, \mathrm{R}^{2}=\mathrm{Me}$

$6 e \mathrm{R}^{1}=4-\mathrm{BrC}_{6} \mathrm{H}_{4} \mathrm{NHCO}, \mathrm{R}^{2}=\mathrm{Me}$

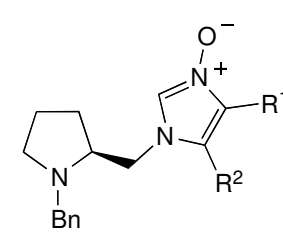

5a-e
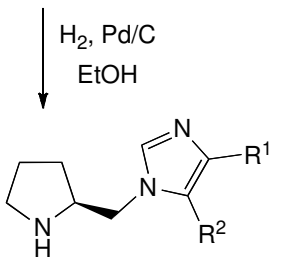

7a,b
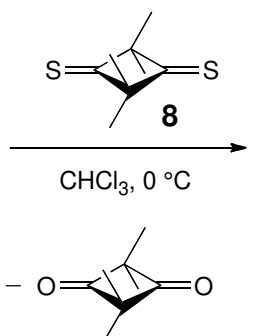

9a $R^{1}=R^{2}=M e$

9b $\mathrm{R}^{1}=\mathrm{R}^{2}=\mathrm{Ph}$

9c $\mathrm{R}^{1}=4-\mathrm{BrC}_{6} \mathrm{H}_{4} \mathrm{NHCO}, \mathrm{R}^{2}=\mathrm{Me}$

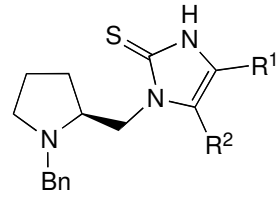$$
\text { Bn }
$$

7a $R^{1}=R^{2}=P h$

7b $\mathrm{R}^{1}=\mathrm{PhNHCO}, \mathrm{R}^{2}=\mathrm{Me}$

\section{Scheme 3}

The 2-unsubstituted imidazole $N$-oxides 5a,c and e reacted with 2,2,4,4tetramethylcyclobutane-1,3-dithione (8) via the so-called 'sulfur-transfer reaction' to give optically active imidazole-2-thiones 9a-c (Scheme 3$).^{20}$ In these reactions, imidazole $\mathrm{N}$-oxides 5 react as 'nitrone-like' 1,3-dipoles, and after the 1,3-dipolar 
cycloaddition, the intermediate 1,4,2-oxathiazolidine undergoes a fragmentation to yield 9 and 2,2,4,4-tetramethyl-3-thioxocyclobutanone. The latter can undergo the 'sulfurtransfer reaction' with a molecule 5 once more, leading to $\mathbf{9}$ and 2,2,4,4tetramethylcyclobutane-1,3-dione. The obtained imidazole-2-thiones 9 are characterized by the thiourea moiety, which is of great importance in asymmetric synthesis and organocatalysis. $^{21}$

The alkylation of imidazoles with long-chain alkyl bromides and subsequent anion exchange is a typical procedure for the preparation of imidazolium salts with the characteristic behavior of ionic liquids (ILs). ${ }^{22}$ Optically active ionic liquids are of special interest, but to date, proline-derived examples are rare. ${ }^{23}$ Thus, imidazoles 6 were alkylated with butyl, hexyl, and octyl bromide in boiling acetonitrile. The obtained imidazolium bromides $\mathbf{1 0}$ dissolved in acetone were treated with an equimolar amount of $\mathrm{NaBF}_{4}$ at room temperature to give the corresponding tetrafluoroborates $\mathbf{1 1}$ (Scheme 4). All of them are liquids at room temperature and, therefore, belong to the class of optically active 'room temperature ionic liquids' (RTILs).

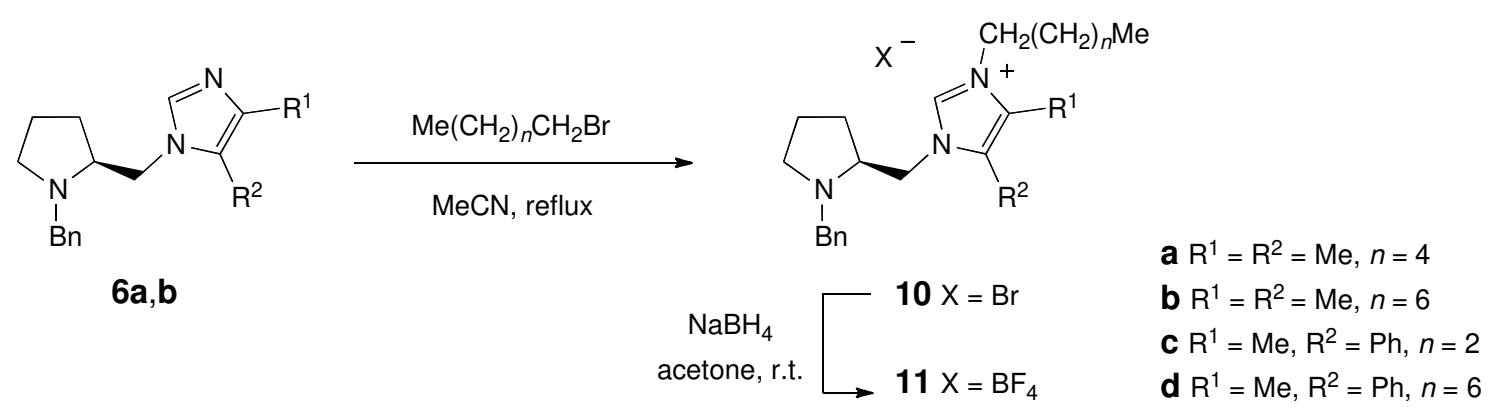

\section{Scheme 4}

In a recent paper, we described the synthesis of a new type of ionic liquids containing a 3 -alkoxyimidazolium cation. ${ }^{8 \mathrm{~d}}$ Similary, the alkylation of imidazole $\mathrm{N}$ oxides 5 were carried out with equimolar amounts of the respective alkyl bromides in $\mathrm{CHCl}_{3}$ solution at room temperature to give imidazolium bromides 12 (Scheme 5). The latter were transformed to the corresponding tetrafluoroborates $\mathbf{1 3}$ in the usual way. It is worth mentioning that under the applied reaction conditions the alkylation of compounds $\mathbf{5}$ and $\mathbf{6}$ occurred selectively at the imidazole fragment. 


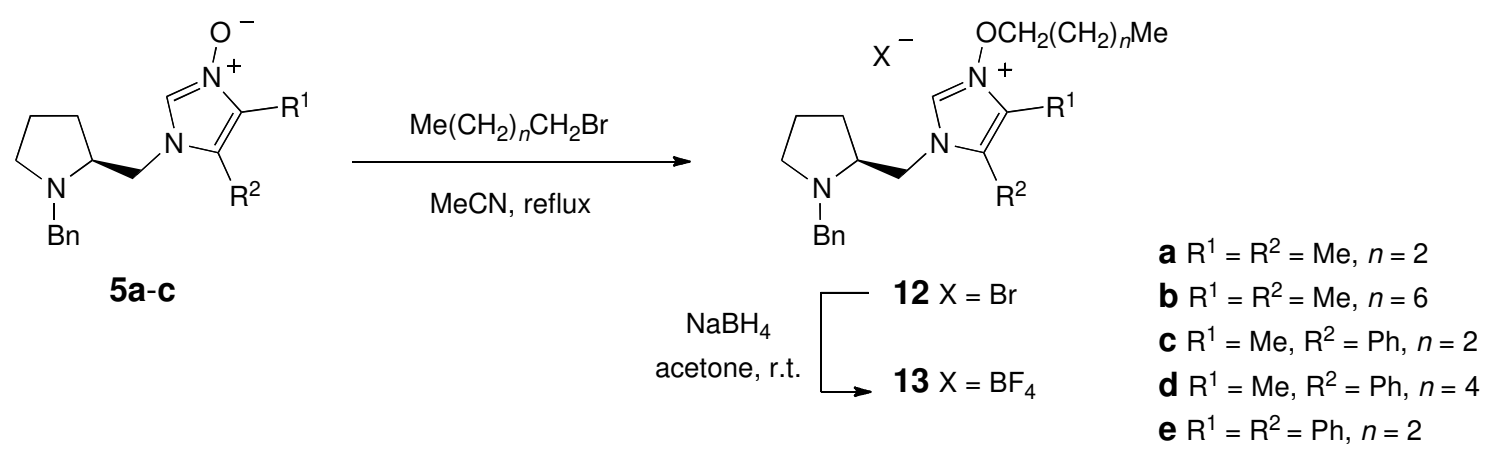

Scheme 5

\section{Conclusions}

The results described in the present paper show that $N$-benzyl L-prolinamine is a useful starting material for the preparation of a new type of optically active 2unsubstituted imidazole $\mathrm{N}$-oxides. Based on the known reactivity of these $\mathrm{N}$-oxides, they can be converted to a variety of other optically active imidazole derivatives such as the parent imidazoles and imidazole-2-thiones. The debenzylation with simultaneous deoxygenation of imidazole $\mathrm{N}$-oxides 5 leading to imidazoles of type 7 can be achieved conveniently by hydrogenolysis under standard conditions. Alkylation of the prepared imidazole $\mathrm{N}$-oxides as well as the corresponding imidazoles leads to $\mathrm{N}$ alkoxyimidazolium and $\mathrm{N}$-alkylimidazolium salts, respectively, with the properties of optically active 'room temperature ionic liquids' (RTILs).

The optically active products described in the present study are of interest as potential ligands for asymmetric synthesis and for organocatalysis. The easily available ionic liquids can be used as reaction media for small-scale organic synthesis.

\section{Experimental}

\subsection{General}

Melting points were determined in a capillary using a Melt-Temp. II apparatus (Aldrich) or STUART SMP30 and are uncorrected. The IR Spectra were recorded on a NEXUS FT-IR spectrophotometer in $\mathrm{KBr}$; absorptions $(v)$ in $\mathrm{cm}^{-1}$. The ${ }^{1} \mathrm{H}$ and ${ }^{13} \mathrm{C}\left\{{ }^{1} \mathrm{H}\right\}$ NMR spectra were measured on a Bruker Avance III instrument (600 and $150 \mathrm{MHz}$, resp.) using solvent signal as reference. Multiplicity of signals in the ${ }^{13} \mathrm{C}$ NMR spectra was established using HMQC technique. Chemical shifts (') are given in ppm and coupling constants $J$ in Hz. Assignments of signals in ${ }^{13} \mathrm{C}$ NMR spectra were made on the basis of HMQC experiments. HR-MS: Bruker maXis spectrometer; ESI-MS: Varian 
500. Optical rotations were determined on a PERKIN-ELMER 241 MC polarimeter for $»=589 \mathrm{~nm}$.

\subsection{Starting Materials}

All solvents and reagents are commercially available and used as received. $(2 S)$ Benzylproline $^{24}$ and \pm -hydroxyimino ketones $4^{20 \mathrm{a}}$ were prepared according to known procedures.

\subsection{1. [(2S)- $N$-Benzylpyrrolidin-2-yl]methanol ( $N$-benzylprolinol)}

A solution of $(2 S)$-benzylproline $(3 \mathrm{~g}, 14.6 \mathrm{mmol})$ in THF $(30 \mathrm{ml})$ was cooled to $0{ }^{\circ} \mathrm{C}$ and a suspension of $\mathrm{LiAlH}_{4}$ in THF (2M, $\left.21.9 \mathrm{mmol}, 12.4 \mathrm{ml}\right)$ was added dropwise. The mixture was stirred overnight at rt. Next, an aqueous solution of $\mathrm{KOH}(10 \%, 15$ $\mathrm{ml})$ was added and the organic product was extracted with $\mathrm{Et}_{2} \mathrm{O}(3 \times 15 \mathrm{ml})$. The organic layers were combined and dried $\left(\mathrm{Na}_{2} \mathrm{SO}_{4}\right)$. After filtration, the solvent was evaporated and the crude, oily product was distilled in a Kugel-Rohr $\left(75-78{ }^{\circ} \mathrm{C}, 0.1 \mathrm{hPa}\right)$. Yield: $2.0 \mathrm{~g}(71 \%)$. Colorless oil. IR (film): v 3405br, 2961m, 2872m, 2799m, 1879w, 1810w, 1721w, 1496m, 1453m, 1211m, 1049w, 1029w, 849m, 839s, 699s. ${ }^{1} \mathrm{H}$ NMR $\left(\mathrm{CDCl}_{3}\right)$ : ' 7.32-7.25 (m, 5H, HC(arom.)); 3.92, 3.32 (AB, $\left.J_{A B}=12.9,2 \mathrm{H}, \mathrm{H}_{2} \mathrm{C}-\mathrm{Ph}\right) ; 3.61$ (dd, $\left.{ }^{2} J_{\mathrm{H}, \mathrm{H}}=10.8,{ }^{3} J_{\mathrm{H}, \mathrm{H}}=3.6,1 \mathrm{H}, \mathrm{H}_{2} \mathrm{C}-\mathrm{OH}\right) ; 3.38\left(\mathrm{dd},{ }^{2} J_{\mathrm{H}, \mathrm{H}}=10.8,{ }^{3} J_{\mathrm{H}, \mathrm{H}}=1.8,1 \mathrm{H}, \mathrm{H}_{2} \mathrm{C}-\right.$ $\mathrm{OH}) ; 2.96-2.90\left(\mathrm{~m}, 1 \mathrm{H}, \mathrm{H}_{2} \mathrm{C}(5)\right) ; 2.72-2.65(\mathrm{~m}, 1 \mathrm{H}, \mathrm{HC}(2)) ; 2.29-2.22(\mathrm{~m}, 1 \mathrm{H}$, $\left.\mathrm{H}_{2} \mathrm{C}(5)\right)$; $1.93-1.85\left(\mathrm{~m}, 1 \mathrm{H}, \mathrm{H}_{2} \mathrm{C}(3)\right)$; 1.82-1.75 (m, 1H, $\left.\mathrm{H}_{2} \mathrm{C}(3)\right) ; 1.71-1.59$ (m, 2H, $\left.\mathrm{H}_{2} \mathrm{C}(4)\right) .{ }^{13} \mathrm{C} \mathrm{NMR}\left(\mathrm{CDCl}_{3}\right)$ : ‘ 139.4 (1 C(arom.)); 128.7, 128.3, 127.1 (5 CH(arom.)); $64.3(\mathrm{C}(2)) ; 61.8\left(\mathrm{CH}_{2}-\mathrm{OH}\right) ; 58.6\left(\mathrm{CH}_{2}-\mathrm{Ph}\right) ; 54.5(\mathrm{C}(5)) ; 27.8(\mathrm{C}(3)) ; 23.5(\mathrm{C}(4))$. $[ \pm]_{\mathrm{D}}^{25}=-58\left(c=1.0, \mathrm{CHCl}_{3}\right) ;$ lit. $^{25}[ \pm]_{\mathrm{D}}{ }^{25}-59.9\left(c=1.0, \mathrm{CHCl}_{3}\right)$.

\subsubsection{1-[(2S)- $N$-Benzylpyrrolidin-2-yl]methanamine 1}

To the solution of triphenylphosphine $(0.86 \mathrm{~g}, 3.3 \mathrm{mmol})$ and phthalimide $(0.48 \mathrm{~g}$, $3.3 \mathrm{mmol})$ in THF (10 ml), $N$-benzylprolinol $(0.57 \mathrm{~g}, 3.0 \mathrm{mmol})$ dissolved in THF (2 $\mathrm{ml}$ ) was added, and the solution was stirred magnetically for $10 \mathrm{~min}$. After this time, diethyl azodicarboxylate (DEAD) (0.62 g, $3.6 \mathrm{mmol})$ was added dropwise, and the mixture obtained was heated at reflux for $8 \mathrm{~h}$. Next, the solvent was removed under reduced pressure, the obtained residue was suspended in $\mathrm{Et}_{2} \mathrm{O}(50 \mathrm{ml})$ and stirred for 1 
$\mathrm{h}$. Then, the precipitate was separated by filtration and the filtrate was removed under reduced pressure. The residue was dissolved in EtOH $(20 \mathrm{ml})$, subsequently hydrazine monohydrate $(0.4 \mathrm{~g}, 8.0 \mathrm{mmol})$ was added, the mixture was heated at reflux for $2 \mathrm{~h}$, and after addition of $\mathrm{HCl}(1 \mathrm{M}, 3 \mathrm{ml})$ heating was continued for $30 \mathrm{~min}$. The precipitate was filtered off and the solvents were removed under reduced pressure. The residue obtained was treated with aqueous $\mathrm{NaOH}(1 \mathrm{M}, 5 \mathrm{ml})$, the organic product was extracted with $\mathrm{Et}_{2} \mathrm{O}(3 \times 25 \mathrm{ml})$, and the combined organic layers were dried $\left(\mathrm{Na}_{2} \mathrm{SO}_{4}\right)$. After filtration, the $\mathrm{Et}_{2} \mathrm{O}$ solution was concentrated under reduced pressure to give $\mathbf{1}$ as a pale yellow oil. The crude product was distilled in a Kugel Rohr $\left(50-52{ }^{\circ} \mathrm{C}, 0.1 \mathrm{hPa}\right)$. Yield: $0.4 \mathrm{~g}$ (70\%). Colorless oil. IR (film): v 3365m, 3289br, 3027m, 2961m, 2871m, 2791m, $1950 \mathrm{~m}, 1877 \mathrm{~m}, 1810 \mathrm{~m}, 1495 \mathrm{~s}, 1453 \mathrm{~s}, 1372 \mathrm{~m}, 1121 \mathrm{~m}, 1072 \mathrm{~m}, 1028 \mathrm{~s}, 910 \mathrm{~m}, 738 \mathrm{~s}$, 699s. ${ }^{1} \mathrm{H} \mathrm{NMR}\left(\mathrm{CDCl}_{3}\right)$ : ' 7.38-7.19 (m, 5H, $\mathrm{HC}($ arom. $\left.)\right) ; 3.96,3.30$ (AB, $J_{A B}=12.9$, $\left.2 \mathrm{H}, \mathrm{H}_{2} \mathrm{C}-\mathrm{Ph}\right) ; 2.97-2.90\left(\mathrm{~m}, 1 \mathrm{H}, \mathrm{H}_{2} \mathrm{C}(5)\right) ; 2.75\left(\mathrm{dd},{ }^{2} J_{\mathrm{H}, \mathrm{H}}=12.9,{ }^{3} J_{\mathrm{H}, \mathrm{H}}=5.4,1 \mathrm{H}, \mathrm{H}_{2} \mathrm{C}-\right.$ $\left.\mathrm{NH}_{2}\right) ; 2.70\left(\mathrm{dd},{ }^{2} J_{\mathrm{H}, \mathrm{H}}=12.9,{ }^{3} J_{\mathrm{H}, \mathrm{H}}=3.6,1 \mathrm{H}, \mathrm{H}_{2} \mathrm{C}-\mathrm{NH}_{2}\right) ; 2.58-2.53(\mathrm{~m}, 1 \mathrm{H}, \mathrm{HC}(2))$; 2.25-2.17 (m, 1H, $\left.\mathrm{H}_{2} \mathrm{C}(5)\right)$; 1.95-1.87 (m, 1H, $\left.\mathrm{H}_{2} \mathrm{C}(3)\right)$; 1.77-1.63 (m, 2H, $\mathrm{H}_{2} \mathrm{C}(4) ; 1 \mathrm{H}$, $\left.\mathrm{H}_{2} \mathrm{C}(3)\right) .{ }^{13} \mathrm{C} \mathrm{NMR}\left(\mathrm{CDCl}_{3}\right)$ : ‘ 139.9 (1 C(arom.)); 128.7, 128.7, 126.8 (5 CH(arom.)); $65.5(\mathrm{C}(2)) ; 59.1\left(\mathrm{CH}_{2}-\mathrm{Ph}\right) ; 54.6(\mathrm{C}(5)) ; 44.1\left(\mathrm{CH}_{2}-\mathrm{NH}_{2}\right) ; 28.0(\mathrm{C}(3)) ; 23.0(\mathrm{C}(4))$ ppm. $[ \pm]_{\mathrm{D}}^{25}=-54\left(c=1.0, \mathrm{CH}_{2} \mathrm{Cl}_{2}\right) ;$ lit. $^{26}[ \pm]_{\mathrm{D}}{ }^{25}=-55\left(c=9.97, \mathrm{CHCl}_{3}\right)$.

\subsubsection{1-[(2S)- $N$-Benzylpyrrolidin-2-yl]- $N$-methylidenemethanamine 2 resp. 3}

To a magnetically stirred solution of $1(1.81 \mathrm{~g}, 9.53 \mathrm{mmol})$ in $\mathrm{MeOH}(10 \mathrm{ml})$, paraformaldehyde $(0.30 \mathrm{~g}, 10 \mathrm{mmol})$ was added, and the mixture was stirred overnight at $\mathrm{rt}$. Then, the solvent was removed under reduced pressure. The oil obtained was used in the next step without purification. Yield: $1.92 \mathrm{~g}$ (98\%). Pale yellow oil. IR (film): $v$ $3361 \mathrm{br}, 3085 \mathrm{~m}, 3061 \mathrm{~m}, 3026 \mathrm{~m}, 2960 \mathrm{~m}, 2872 \mathrm{~m}, 2788 \mathrm{~m}, 1946 \mathrm{~m}, 1875 \mathrm{~m}, 1808 \mathrm{~m}$, $1604 \mathrm{~m}, 1495 \mathrm{~s}, 1453 \mathrm{~s}, 1604 \mathrm{~m}, 1155 \mathrm{w}, 1028 \mathrm{~m}, 916 \mathrm{~m}, 735 \mathrm{~s}, 698 \mathrm{~s} .{ }^{1} \mathrm{H}$ NMR $\left(\mathrm{CDCl}_{3}\right)$ : 7.35-7.16 (m, 5H, HC(arom.)); 4.16, $3.23\left(\mathrm{AB}, J_{A B}=13.2,2 \mathrm{H}, \mathrm{H}_{2} \mathrm{C}-\mathrm{Ph}\right) ; 3.47-3.32$ (br, $\left.2 \mathrm{H}, \mathrm{N}-\mathrm{CH}_{2}-\mathrm{N}\right) ; 2.93-2.87(\mathrm{~m}, 1 \mathrm{H}, \mathrm{HC}(5)) ; 2.68\left(\mathrm{dd},{ }^{2} J_{\mathrm{H}, \mathrm{H}}=12.9,{ }^{3} J_{\mathrm{H}, \mathrm{H}}=4.8,1 \mathrm{H}\right.$, $\left.\mathrm{H}_{2} \mathrm{C}-\mathrm{N}\right) ; 2.57-2.52(\mathrm{~m}, 1 \mathrm{H}, \mathrm{HC}(2)) ; 2.42\left(\mathrm{dd},{ }^{2} J_{\mathrm{H}, \mathrm{H}}=12.9,{ }^{3} J_{\mathrm{H}, \mathrm{H}}=6.6,1 \mathrm{H}, \mathrm{H}_{2} \mathrm{C}-\mathrm{N}\right)$; 2.15-2.09 (m, 1H, HC(5)); 1.98-1.88 (m, 1H, HC(3)); 1.75-1.60 (m, 2H, $\mathrm{H}_{2} \mathrm{C}(4) ; 1 \mathrm{H}$, $\left.\mathrm{H}_{2} \mathrm{C}(3)\right) .{ }^{13} \mathrm{C} \mathrm{NMR}\left(\mathrm{CDCl}_{3}\right)$ : ' 139.9 (C(arom.)); 128.9, 128.2, 126.7 (5, $\mathrm{CH}$ (arom.)); $75.8\left(\mathrm{~N}-\mathrm{CH}_{2}-\mathrm{N}\right) ; 62.4(\mathrm{C}(2)) ; 59.6\left(\mathrm{CH}_{2}-\mathrm{Ph}\right) ; 57.6\left(\mathrm{CH}_{2}-\mathrm{N}\right) ; 54.2(\mathrm{C}(5)) ; 30.2(\mathrm{C}(3))$; $22.6(\mathrm{C}(4))$. ESI-MS: $m / z 203\left(100,[M+1]^{+}\right) .[ \pm]_{\mathrm{D}}{ }^{25}=-14\left(c=1.0, \mathrm{CH}_{2} \mathrm{Cl}_{2}\right)$. 


\subsubsection{Synthesis of imidazole $N$-oxides 5}

Procedure A: A solution of 2 (10 $\mathrm{mmol})$ and the corresponding \pm -hydroxyimino ketone 4 (10 mmol) in EtOH (5 ml) was heated at reflux for $3 \mathrm{~h}$. Next, the solvent was removed under reduced pressure, and the resulting residue was purified by column chromatography or by crystallization.

Procedure B: A mixture of $2(10 \mathrm{mmol})$ and of the corresponding $4(10 \mathrm{mmol})$ in glacial acetic acid $(15 \mathrm{ml})$ was magnetically stirred overnight at $\mathrm{rt}$. Then, $\mathrm{HCl}$ gas was bubbled through the mixture for $1 \mathrm{~h}$. Next, $\mathrm{Et}_{2} \mathrm{O}$ (ca. $100 \mathrm{ml}$ ) was added and the colorless precipitated hydrochloride was filtered, washed with cold $\mathrm{Et}_{2} \mathrm{O}(3 \times 20 \mathrm{ml})$, and dried under reduced pressure. The crude hydrochloride was dissolved in $\mathrm{CH}_{2} \mathrm{Cl}_{2}(25$ $\mathrm{ml}$ ), solid $\mathrm{NaHCO}_{3}(1.5 \mathrm{~g}$ ) was added, and stirring was continued for $1 \mathrm{~h}$ until evolution of $\mathrm{CO}_{2}$ ceased. The inorganic salts were filtered off and the solvent was removed. The crude products 5a-e were purified by column chromatography or by crystallization.

\subsubsection{1- $\{[(2 S)-N$-Benzylpyrrolidin-2-yl]methyl $\}-4,5$-dimethyl-1H-imidazole 3 -} oxide 5a

Yield: 2.5 g (88\%). Colorless oil $\left(\mathrm{SiO}_{2}, \mathrm{MeOH} / \mathrm{AcOEt}, 1: 1\right)$. IR (film): v 35843028br, 2954m, 2926m, 2807m, 1627m, 1495m, 1453s, 1381m, 1338m, 1148m, 1041s, 843w, 733w, 702s. ${ }^{1} \mathrm{H}$ NMR $\left(\mathrm{CDCl}_{3}\right)$ : 7.94 (s, 1H, $\left.\mathrm{HC}(2)\right) ; 7.35-7.21$ (m, 5H, $\mathrm{HC}$ (arom.)); 3.75, $3.54\left(\mathrm{AB}, J_{A B}=12.6,2 \mathrm{H}, \mathrm{H}_{2} \mathrm{C}-\mathrm{Ph}\right) ; 3.68\left(\mathrm{dd},{ }^{2} J_{\mathrm{H}, \mathrm{H}}=14.4,{ }^{3} J_{\mathrm{H}, \mathrm{H}}=\right.$ $\left.4.8,1 \mathrm{H}, \mathrm{H}_{2} \mathrm{C}-\mathrm{N}\right) ; 3.60\left(\mathrm{dd},{ }^{2} J_{\mathrm{H}, \mathrm{H}}=14.4,{ }^{3} J_{\mathrm{H}, \mathrm{H}}=6.0,1 \mathrm{H}, \mathrm{H}_{2} \mathrm{C}-\mathrm{N}\right) ; 3.04-2.99(\mathrm{~m}, 1 \mathrm{H}$, $\left.\mathrm{H}_{2} \mathrm{C}\left(5^{\prime}\right)\right)$; 2.92-2.86 (m, 1H, $\left.\mathrm{HC}\left(2^{\prime}\right)\right)$; 2.39-2.32 (m, 1H, $\left.\mathrm{H}_{2} \mathrm{C}\left(5^{\prime}\right)\right)$; 2.15 (s, 3H, $\mathrm{H}_{3} \mathrm{C}-$ $\mathrm{C}(4)) ; 2.05\left(\mathrm{~s}, 3 \mathrm{H}, \mathrm{H}_{3} \mathrm{C}-\mathrm{C}(5)\right) ; 1.93-1.85\left(\mathrm{~m}, 1 \mathrm{H}, \mathrm{H}_{2} \mathrm{C}\left(3^{\prime}\right)\right) ; 1.75-1.69(\mathrm{~m}, 1 \mathrm{H}$, $\left.\mathrm{H}_{2} \mathrm{C}\left(4^{\prime}\right)\right)$; $1.68-1.59$ (m, $\left.1 \mathrm{H}, \mathrm{H}_{2} \mathrm{C}\left(4^{\prime}\right)\right)$; $1.49-1.43$ (m, 1H, $\left.\mathrm{H}_{2} \mathrm{C}\left(3^{\prime}\right)\right) .{ }^{13} \mathrm{C} \mathrm{NMR}\left(\mathrm{CDCl}_{3}\right)$ : ' 138.9 (C(arom.)); 128.8, 128.5, 127.3 (5 CH(arom.)); 126.3 (1 C(imid.)); 124.9 (HC(2)); 121.0 (1 C(imid.)); 63.2 (HC(2')); $60.1\left(\mathrm{CH}_{2}-\mathrm{Ph}\right) ; 54.8$ (C(5')); $49.1\left(\mathrm{CH}_{2}-\right.$ $\mathrm{N}) ; 28.5\left(\mathrm{C}\left(3^{\prime}\right)\right) ; 23.3\left(\mathrm{C}\left(4^{\prime}\right)\right) ; 8.9\left(\mathrm{CH}_{3}-\mathrm{C}(4)\right)$; $7.3\left(\mathrm{CH}_{3}-\mathrm{C}(5)\right)$. HR-ESI-MS (MeOH + $\mathrm{HCOOH}$ ): 286.1909 (calcd 286.1914 for $\left.\mathrm{C}_{17} \mathrm{H}_{24} \mathrm{~N}_{3} \mathrm{O},[M+1]^{+}\right) .[ \pm]_{\mathrm{D}}^{25}=-18(c=1.0$, $\mathrm{CH}_{2} \mathrm{Cl}_{2}$ ).

4.2.4.2. $1-\{[(2 S)-N$-Benzylpyrrolidin-2-yl]methyl $\}-4-$-methyl-5-phenyl- $1 H$-imidazole 3-oxide 5b 
Yield: 1.3 g, 40\% (method A), 2.7 g, 80\% (method B). Colorless oil $\left(\mathrm{SiO}_{2}\right.$, MeOH/AcOEt, 1:1). IR (film): v 3647-3028br, 2962m, 2876m, 2803m, 1683s, 1551m, 1496s, 1452m, 1381m, 1028m, 922w, 847w, 764m, 734m, 702m, 642s. ${ }^{1} \mathrm{H}$ NMR $\left(\mathrm{CDCl}_{3}\right)$ : ‘ 8.29 (s, 1H, HC(2)); 7.49-7.45 (m, 3H, HC(arom.)); 7.32-7.29 (m, 2H, HC(arom.)); 7.26-7.24 (m, 3H, HC(arom.)); 7.23-7.20 (m, 2H, HC(arom.)); 3.77 (dd, $\left.J_{\mathrm{H}, \mathrm{H}}=14.4, J_{\mathrm{H}, \mathrm{H}}=4.8,1 \mathrm{H}, \mathrm{H}_{2} \mathrm{C}-\mathrm{N}\right) ; 3.64-3.58\left(\mathrm{~m}, 1 \mathrm{H}, \mathrm{H}_{2} \mathrm{C}-\mathrm{Ph}, 1 \mathrm{H}, \mathrm{H}_{2} \mathrm{C}-\mathrm{N}\right) ; 3.38$ $\left(\mathrm{AB}, J_{A B}=12.9,2 \mathrm{H}, \mathrm{H}_{2} \mathrm{C}-\mathrm{Ph}\right) ; 2.96-2.91\left(\mathrm{~m}, 1 \mathrm{H}, \mathrm{H}_{2} \mathrm{C}\left(5^{\prime}\right)\right) ; 2.71-2.68(\mathrm{~m}, 1 \mathrm{H}$, $\left.\mathrm{H}_{2} \mathrm{C}\left(2^{\prime}\right)\right) ; 2.28-2.22\left(\mathrm{~m}, 1 \mathrm{H}, \mathrm{H}_{2} \mathrm{C}\left(5^{\prime}\right)\right) ; 2.19$ (s, 3H, $\left.\mathrm{H}_{3} \mathrm{C}-\mathrm{C}(4)\right) ; 1.75-1.73$ (m, $1 \mathrm{H}$, $\left.\mathrm{H}_{2} \mathrm{C}\left(3^{\prime}\right)\right)$; $1.63-1.60$ (m, $\left.1 \mathrm{H}, \mathrm{H}_{2} \mathrm{C}\left(4^{\prime}\right)\right)$; $1.53-1.48$ (m, $\left.1 \mathrm{H}, \mathrm{H}_{2} \mathrm{C}\left(4^{\prime}\right)\right)$; $1.33-1.29$ (m, $1 \mathrm{H}$, $\left.\mathrm{H}_{2} \mathrm{C}\left(3^{\prime}\right)\right) .{ }^{13} \mathrm{C} \mathrm{NMR}\left(\mathrm{CDCl}_{3}\right)$ : ' 138.3 (1 C(arom.)); 130.7, 129.8, 129.4, 129.1, 129.1, 128.6, 127.5, 127.2 (9 signals for $10 \mathrm{CH}$ (arom.), 2 C(arom.), $1 \mathrm{HC}(2), 2 \mathrm{C}$ (imid.)); 63.4 $\left.\left(\mathrm{HC}\left(2^{\prime}\right)\right) ; 59.7\left(\mathrm{CH}_{2}-\mathrm{Ph}\right) ; 54.5\left(\mathrm{C}\left(5^{\prime}\right)\right) ; 49.5\left(\mathrm{CH}_{2}-\mathrm{N}\right) ; 28.5\left(\mathrm{C}\left(3^{\prime}\right)\right) ; 23.3\left(\mathrm{C}^{\prime} 4^{\prime}\right)\right) ; 7.9$ $\left(\mathrm{H}_{3} \mathrm{C}-\mathrm{C}(4)\right)$. HR-ESI-MS (MeOH + $\left.\mathrm{HCOOH}\right): 348.2068$ (calcd 348.2070 for $\left.\mathrm{C}_{22} \mathrm{H}_{26} \mathrm{~N}_{3} \mathrm{O},[M+1]^{+}\right) .[ \pm]_{\mathrm{D}}^{25}=-24\left(c=1.0, \mathrm{CH}_{2} \mathrm{Cl}_{2}\right)$.

\subsubsection{3. $1-\{[(2 S)-N$-Benzylpyrrolidin-2-yl]methyl $\}-4,5$-diphenyl-1H-imidazole 3 - oxide $5 \mathrm{c}$}

Yield: $2.6 \mathrm{~g}, 65 \%(\operatorname{method} \mathrm{A}), 3.1 \mathrm{~g}, 75 \%(\operatorname{method} \mathrm{B})$. Colorless crystals. Mp $160-161{ }^{\circ} \mathrm{C}\left(\mathrm{CHCl}_{3} / \mathrm{Et}_{2} \mathrm{O}\right)$. IR (KBr): v 3646-3027br, 2961m, 2876w, 2810m, 1736m, $1604 \mathrm{~m}, 1495 \mathrm{~m}, 1445 \mathrm{~m}, 1346 \mathrm{~m}, 1193 \mathrm{~m}, 1076 \mathrm{~m}, 1039 \mathrm{~s}, 918 \mathrm{~m}, 864 \mathrm{~s}, 762 \mathrm{~s}, 700 \mathrm{~s}, 656 \mathrm{~m}$. ${ }^{1} \mathrm{H}$ NMR $\left(\mathrm{CDCl}_{3}\right)$ : ' 8.35 (s, 1H, $\left.\mathrm{HC}(2)\right)$; 7.55-7.52 (m, 2H, HC(arom.)); 7.43-7.36 (m, 3H, HC(arom.)); 7.32-7.28 (m, 2H, HC(arom.)); 7.27-7.19 (m, 8H, HC(arom.)); 3.75 $\left(\mathrm{dd},{ }^{2} J_{\mathrm{H}, \mathrm{H}}=14.0,{ }^{3} J_{\mathrm{H}, \mathrm{H}}=4.2,1 \mathrm{H}, \mathrm{H}_{2} \mathrm{C}-\mathrm{N}\right) ; 3.62\left(\mathrm{dd},{ }^{2} J_{\mathrm{H}, \mathrm{H}}=14.0,{ }^{3} J_{\mathrm{H}, \mathrm{H}}=6.0,1 \mathrm{H}, \mathrm{H}_{2} \mathrm{C}-\right.$ $\mathrm{N})$; 3.63, $3.39\left(\mathrm{AB}, J_{A B}=13.2,2 \mathrm{H}, \mathrm{H}_{2} \mathrm{C}-\mathrm{Ph}\right) ; 2.98-2.93\left(\mathrm{~m}, 1 \mathrm{H}, \mathrm{H}_{2} \mathrm{C}\left(5^{\prime}\right)\right)$; 2.79-2.72 (m, 1H, $\left.\mathrm{HC}\left(2^{\prime}\right)\right)$; 2.31-2.24 (m, 1H, $\left.\mathrm{H}_{2} \mathrm{C}\left(5^{\prime}\right)\right)$; 1.84-1.76 (m, 1H, $\left.\mathrm{H}_{2} \mathrm{C}\left(3^{\prime}\right)\right)$; 1.69-1.62 (m, 1H, $\left.\mathrm{H}_{2} \mathrm{C}\left(4^{\prime}\right)\right)$; 1.60-1.51 (m, 1H, $\left.\mathrm{H}_{2} \mathrm{C}\left(4^{\prime}\right)\right)$; $1.43-1.37$ (m, 1H, $\left.\mathrm{H}_{2} \mathrm{C}\left(3^{\prime}\right)\right)$. ${ }^{13} \mathrm{C} \mathrm{NMR}$ $\left(\mathrm{CDCl}_{3}\right)$ : ‘ 138.7 (1 C(arom.)); 131.0, 130.2, 129.6, 129.5, 129.1, 128.8, 128.5, 128.1, 128.0, 127.7, 127.3, 127.3, 126.7 (2 C(arom.), 15 HC(arom.), 2 C(imid.)); 126.9 (HC(2)) $63.1\left(\mathrm{HC}\left(2^{\prime}\right)\right) ; 59.6\left(\mathrm{CH}_{2}-\mathrm{Ph}\right) ; 54.4\left(\mathrm{C}\left(5^{\prime}\right)\right) ; 49.1\left(\mathrm{CH}_{2}-\mathrm{N}\right) ; 28.4\left(\mathrm{C}\left(4^{\prime}\right)\right) ; 23.2$ $\left(\mathrm{C}\left(3^{\prime}\right)\right)$. ESI HR-ESI-MS $(\mathrm{MeOH}+\mathrm{HCOOH}): 410.2228$ (calcd 410.2227 for $\left.\mathrm{C}_{27} \mathrm{H}_{28} \mathrm{~N}_{3} \mathrm{O},[M+1]^{+}\right) .[ \pm]_{\mathrm{D}}^{25}=-5\left(c=1.0, \mathrm{CH}_{2} \mathrm{Cl}_{2}\right)$.

4.2.4.4. $N$-Phenyl-1-\{[(2S)-N-benzylpyrrolidin-2-yl]methyl $\}-5-$ methyl-1Himidazole-4-carboxamide 3-oxide 5d 
Yield: $3.0 \mathrm{~g}(78 \%)$. Colorless crystals. $\mathrm{Mp} 120-122{ }^{\circ} \mathrm{C}\left(\mathrm{Et}_{2} \mathrm{O}\right)$. IR (KBr): $v$ 3427br, 3171m, 3062m, 2978w, 2921w, 2798m, 1672s, 1619m, 1598m, 1560s, 1310s, 1273s, 759m, 750m, 701m, 630m. ${ }^{1} \mathrm{H}$ NMR $\left(\mathrm{CDCl}_{3}\right)$ : ' 12.98 (s, 1H, NH); 7.95 (s, 1H, $\mathrm{HC}(2))$; 7.74-7.69 (m, 2H, HC(arom.)); 7.36-7.31 (m, 4H, HC(arom.)); 7.34-7.24 (m, $4 \mathrm{H}, \mathrm{HC}($ arom. $)) ; 3.76,3.63\left(\mathrm{AB}, J_{A B}=13.6,2 \mathrm{H}, \mathrm{H}_{2} \mathrm{C}-\mathrm{Ph}\right) ; 3.76\left(\mathrm{dd},{ }^{2} J_{\mathrm{H}, \mathrm{H}}=14.4,{ }^{3} J_{\mathrm{H}, \mathrm{H}}\right.$ $\left.=4.8,1 \mathrm{H}, \mathrm{H}_{2} \mathrm{C}-\mathrm{N}\right) ; 3.66\left(\mathrm{dd},{ }^{2} J_{\mathrm{H}, \mathrm{H}}=14.4,{ }^{3} J_{\mathrm{H}, \mathrm{H}}=5.40 \mathrm{~Hz}, 1 \mathrm{H}, \mathrm{H}_{2} \mathrm{C}-\mathrm{N}\right) ; 3.10-3.04(\mathrm{~m}$, $\left.1 \mathrm{H}, \mathrm{H}_{2} \mathrm{C}^{\prime}(5)\right)$; 3.02-2.96 (m, 1H, $\left.\mathrm{H}_{2} \mathrm{C}^{\prime}(2)\right) ; 2.58$ (s, 3H, $\left.\mathrm{H}_{3} \mathrm{C}-\mathrm{C}(5)\right)$; 2.47-2.40 (m, 1H, $\left.\mathrm{H}_{2} \mathrm{C}\left(5^{\prime}\right)\right) ; 2.00-1.91$ (m, 1H, $\left.\mathrm{H}_{2} \mathrm{C}\left(3^{\prime}\right)\right) ; 1.80-1.73$ (m, 1H, $\left.\mathrm{H}_{2} \mathrm{C}\left(4^{\prime}\right)\right)$; 1.65-1.56 (m, 1H, $\left.\mathrm{H}_{2} \mathrm{C}\left(4^{\prime}\right)\right)$; 1.48-1.41 (m, 1H, $\left.\mathrm{H}_{2} \mathrm{C}\left(3^{\prime}\right)\right) .{ }^{13} \mathrm{C} \mathrm{NMR}\left(\mathrm{CDCl}_{3}\right)$ : ' $157.9(\mathrm{C}=\mathrm{O}) ; 138.7,138.4$ (2 C(arom.)); 131.5, 129.1, 129.0, 128.8, 127.7, 124.2, 121.7, 120.1 (10 CH(arom.), 2 C(imid.)); 126.0 (HC(2)); 62.8 ( $\left.\mathrm{HC}\left(2^{\prime}\right)\right)$; $60.3\left(\mathrm{CH}_{2}-\mathrm{Ph}\right) ; 55.0\left(\mathrm{C}\left(5^{\prime}\right)\right) ; 48.7\left(\mathrm{CH}_{2}-\mathrm{N}\right)$; $28.4\left(\mathrm{C}\left(3^{\prime}\right)\right) ; 23.7\left(\mathrm{C}\left(4^{\prime}\right)\right) ; 10.0\left(\mathrm{H}_{3} \mathrm{C}-\mathrm{C}(5)\right)$. HR-ESI-MS $(\mathrm{MeOH}+\mathrm{HCOOH})$ : 391.2124 (calcd 391.2129 for $\left.\mathrm{C}_{23} \mathrm{H}_{27} \mathrm{~N}_{4} \mathrm{O}_{2},[M+1]^{+}\right)$. [ $]_{\mathrm{D}}{ }^{25}=-27\left(c=1.0, \mathrm{CH}_{2} \mathrm{Cl}_{2}\right)$.

\subsubsection{5. $N$-(4-Bromophenyl)-1- $\{[(2 S)-N$-benzylpyrrolidin-2-yl $]$ methyl $\}-5$-methyl- $1 H$-imidazole-4-carboxamide 3-oxide $5 \mathrm{e}$}

Yield: $3.8 \mathrm{~g}(81 \%)$. Colorless crystals. Mp $168-170{ }^{\circ} \mathrm{C}$ (Acetone). IR (KBr): $v$ 3432br, 3077m, 2975w, 2939w, 2919w, 2802m, 1675s, 1612s, 1556s, 1488s, 1309m, 1069m, 817m, 757m, 702s, 636m, 503m. ${ }^{1} \mathrm{H} \mathrm{NMR}\left(\mathrm{CDCl}_{3}\right)$ : ' 13.00 (s, 1H, NH); 7.91 (s, 1H, HC(2)); 7.63-7.59 (m, 2H, HC(arom.)); 7.45-7.41 (m, 2H, HC(arom.)); 7.357.31 (m, 2H, HC(arom.)); 7.29-7.25 (m, 3H, HC(arom.)); 3.65, 3.54 (AB, $J_{A B}=12.6$,

$\left.2 \mathrm{H}, \mathrm{H}_{2} \mathrm{C}-\mathrm{Ph}\right) ; 3.67\left(\mathrm{dd},{ }^{2} J_{\mathrm{H}, \mathrm{H}}=14.4,{ }^{3} J_{\mathrm{H}, \mathrm{H}}=4.8,1 \mathrm{H}, \mathrm{H}_{2} \mathrm{C}-\mathrm{N}\right) ; 3.57\left(\mathrm{dd},{ }^{2} J_{\mathrm{H}, \mathrm{H}}=14.4\right.$, $\left.{ }^{3} J_{\mathrm{H}, \mathrm{H}}=5.4,1 \mathrm{H}, \mathrm{H}_{2} \mathrm{C}-\mathrm{N}\right) ; 3.09-3.03\left(\mathrm{~m}, 1 \mathrm{H}, \mathrm{H}_{2} \mathrm{C}\left(5^{\prime}\right)\right)$; 3.02-2.95 (m, $\left.1 \mathrm{H}, \mathrm{H}_{2} \mathrm{C}\left(2^{\prime}\right)\right)$; 2.55 (s, 3H, $\left.\mathrm{H}_{3} \mathrm{C}-\mathrm{C}(5)\right)$; 2.47-2.40 (m, 1H, $\mathrm{H}_{2} \mathrm{C}\left(5^{\prime}\right)$ ); 2.00-1.91 (m, 1H, $\mathrm{H}_{2} \mathrm{C}\left(3^{\prime}\right)$ ); 1.80-1.72 (m, 1H, $\mathrm{H}_{2} \mathrm{C}\left(4^{\prime}\right)$ ); 1.64-1.54 (m, 1H, $\mathrm{H}_{2} \mathrm{C}\left(4^{\prime}\right)$ ); 1.49-1.39 (m, 1H, $\mathrm{H}_{2} \mathrm{C}\left(3^{\prime}\right)$ ). ${ }^{13} \mathrm{C} \mathrm{NMR}\left(\mathrm{CDCl}_{3}\right)$ : ' $157.9(\mathrm{C}=\mathrm{O}) ; 138.7,137.5$ (2 C(arom.)); 132.0, 131.7, 129.0, 128.8, 127.8, 122.2, 121.5, 116.7 (1 C(arom.), 9 CH(arom.), 2 C(imid.)); 126.1 (HC(2)); 62.8 (HC(2’)); $60.3\left(\mathrm{CH}_{2}-\mathrm{Ph}\right) ; 55.1\left(\mathrm{C}\left(5^{\prime}\right)\right) ; 48.8\left(\mathrm{CH}_{2}-\mathrm{N}\right) ; 28.4\left(\mathrm{C}\left(3^{\prime}\right)\right) ; 23.7$ $\left(\mathrm{C}\left(4^{\prime}\right)\right) ; 10.0\left(\mathrm{H}_{3} \mathrm{C}-\mathrm{C}(5)\right)$. HR-ESI-MS (MeOH + HCOOH): 469.1230 (calcd 469.1234 for $\left.\mathrm{C}_{23} \mathrm{H}_{26} \mathrm{BrN}_{4} \mathrm{O}_{2},\left[M\left({ }^{79} \mathrm{Br}\right)+1\right]^{+}\right) .[ \pm]_{\mathrm{D}}{ }^{25}=-22\left(c=1.0, \mathrm{CH}_{2} \mathrm{Cl}_{2}\right)$.

\subsubsection{Synthesis of imidazoles 6}


To a magnetically stirred solution of an imidazole $N$-oxide $5(5 \mathrm{mmol})$ in $\mathrm{EtOH}$ $(15 \mathrm{ml})$, a suspension of freshly prepared Raney-Nickel in EtOH was added in small portions at rt. The progress of the reaction was followed by TLC $\left(\mathrm{SiO}_{2}, \mathrm{MeOH} / \mathrm{AcOEt}\right.$ 1:1). Then, the mixture was filtered and the filtrate was concentrated under reduced pressure. The crude products 6a-e were purified by chromatography or by crystallization.

\subsubsection{1- $\{[(2 S)-N$-Benzylpyrrolidin-2-yl]methyl $\}-4,5$-dimethyl-1H-imidazole $6 a$}

Yield: $1.0 \mathrm{~g}(75 \%)$. Pale yellow oil $\left(\mathrm{SiO}_{2}, \mathrm{MeOH} / \mathrm{AcOEt}, 1: 1\right)$. IR (film): $v$ 3428br, 2926m, 2854w, 2360w, 2342w, 1637w, 1560m, 1416m, 1245m, 1216m, 1045w, 1021w, 801m, 748m, 700m. ${ }^{1} \mathrm{H}$ NMR $\left(\mathrm{CDCl}_{3}\right)$ : ' 7.40 (s, 1H, HC(2)); 7.337.29 (m, 4H, HC(arom.)); 7.26-7.21 (m, 1H, HC(arom.)); $3.76\left(\mathrm{dd},{ }^{2} J_{\mathrm{H}, \mathrm{H}}=14.4,{ }^{3} J_{\mathrm{H}, \mathrm{H}}=\right.$ $\left.4.8,1 \mathrm{H}, \mathrm{H}_{2} \mathrm{C}-\mathrm{N}\right) ; 3.76,3.44\left(\mathrm{AB}, J_{A B}=13.2,2 \mathrm{H}, \mathrm{H}_{2} \mathrm{C}-\mathrm{Ph}\right) ; 3.66\left(\mathrm{dd},{ }^{2} J_{\mathrm{H}, \mathrm{H}}=14.4,{ }^{3} J_{\mathrm{H}, \mathrm{H}}\right.$ = 7.2, 1H, $\left.\mathrm{H}_{2} \mathrm{C}-\mathrm{N}\right) ; 3.31-2.96\left(\mathrm{~m}, 1 \mathrm{H}, \mathrm{H}_{2} \mathrm{C}\left(5^{\prime}\right)\right) ; 2.87-2.82\left(\mathrm{~m}, 1 \mathrm{H}, \mathrm{H}_{2} \mathrm{C}\left(2^{\prime}\right)\right) ; 2.33-$ 2.27 (m, 1H, $\mathrm{H}_{2} \mathrm{C}\left(5^{\prime}\right)$ ); 2.13 (s, 3H, $\left.\mathrm{H}_{3} \mathrm{C}-\mathrm{C}(4)\right)$ ) 2.06 (s, 3H, $\mathrm{H}_{3} \mathrm{C}-\mathrm{C}(5)$ ); 1.89-1.81 (m, $\left.1 \mathrm{H}, \mathrm{H}_{2} \mathrm{C}\left(3^{\prime}\right)\right)$; 1.73-1.65 (m, 2H, $\left.\mathrm{H}_{2} \mathrm{C}\left(4^{\prime}\right)\right)$; $1.57-1.49$ (m, $\left.1 \mathrm{H}, \mathrm{H}_{2} \mathrm{C}\left(3^{\prime}\right)\right)$ ). ${ }^{13} \mathrm{C} \mathrm{NMR}$ $\left(\mathrm{CDCl}_{3}\right)$ : ' 139.2 (1 C(arom.)); 132.0 (HC(2)); 129.8, 129.0, 126.7, 121.1, 120.2 (5 $\mathrm{CH}$ (arom.); 2 C(imid.)); 63.5 (HC(2')); $59.1\left(\mathrm{CH}_{2}-\mathrm{Ph}\right) ; 54.7\left(\mathrm{C}\left(5^{\prime}\right)\right) ; 49.6\left(\mathrm{CH}_{2}-\mathrm{N}\right)$; $29.0\left(\mathrm{C}\left(3^{\prime}\right)\right)$; $23.3\left(\mathrm{C}\left(4^{\prime}\right)\right)$; $8.8\left(\mathrm{H}_{3} \mathrm{C}-\mathrm{C}(4)\right)$; $7.2\left(\mathrm{H}_{3} \mathrm{C}-\mathrm{C}(5)\right)$ ppm. HR-ESI-MS (MeOH $+\mathrm{HCOOH}): 270.1961$ (calcd 270.1965 for $\left.\mathrm{C}_{17} \mathrm{H}_{24} \mathrm{~N}_{3},[M+1]^{+}\right) \cdot[ \pm]_{\mathrm{D}}{ }^{25}=-25(c=1.0$, $\mathrm{CH}_{2} \mathrm{Cl}_{2}$ ).

\subsubsection{1- $\{[(2 S)-N$-Benzylpyrrolidin-2-yl]methyl $\}-4-m e t h y l-5-p h e n y l-1 H$-imidazole} 6b

Yield: 1.2 g (70\%). Pale yellow oil $\left(\mathrm{SiO}_{2}, \mathrm{MeOH} / \mathrm{AcOEt}, 1: 1\right)$. IR (film): v 3583$3369 \mathrm{br}, 3059 \mathrm{~m}, 3029 \mathrm{~m}, 2948 \mathrm{~m}, 2873 \mathrm{~m}, 2796 \mathrm{~m}, 1955 \mathrm{~m}, 1888 \mathrm{~m}, 1815 \mathrm{~m}, 1688 \mathrm{~m}$, 1605w, 1493s, 1452m, 1207m, 1124m, 1029m, 966m, 701m. ${ }^{1} \mathrm{H}$ NMR $\left(\mathrm{CDCl}_{3}\right){ }^{\prime}{ }^{\prime} 7.58$ (s, 1H, HC(2)); 7.46-7.41 (m, 2H, HC(arom.)); 7.39-7.35 (m, 1H, HC(arom.)); 7.30$7.26(\mathrm{~m}, 4 \mathrm{H}, \mathrm{HC}(\operatorname{arom}).) ; 7.24-7.20(\mathrm{~m}, 1 \mathrm{H}, \mathrm{HC}(\operatorname{arom}).) ; 7.19-7.17$ (m, 2H, $\mathrm{HC}$ (arom.)); 3.92 (dd, $\left.{ }^{2} J_{\mathrm{H}, \mathrm{H}}=14.4,{ }^{3} J_{\mathrm{H}, \mathrm{H}}=4.8,1 \mathrm{H}, \mathrm{H}_{2} \mathrm{C}-\mathrm{N}\right) ; 3.76\left(\mathrm{dd},{ }^{2} J_{\mathrm{H}, \mathrm{H}}=14.4\right.$, $\left.{ }^{3} J_{\mathrm{H}, \mathrm{H}}=7.2,1 \mathrm{H}, \mathrm{H}_{2} \mathrm{C}-\mathrm{N}\right) ; 3.55,3.19\left(\mathrm{AB}, J_{A B}=13.2,2 \mathrm{H}, \mathrm{H}_{2} \mathrm{C}-\mathrm{Ph} ; 2.89-2.85(\mathrm{~m}, 1 \mathrm{H}\right.$, $\left.\mathrm{H}_{2} \mathrm{C}\left(5^{\prime}\right)\right)$; 2.63-2.57 (m, 1H, $\left.\mathrm{HC}\left(2^{\prime}\right)\right) ; 2.18$ (s, 3H, $\left.\mathrm{H}_{3} \mathrm{C}-\mathrm{C}(4)\right)$; 2.18-2.13 (m, 1H, $\left.\mathrm{H}_{2} \mathrm{C}\left(5^{\prime}\right)\right)$; 1.73-1.65 (m, 1H, $\left.\mathrm{H}_{2} \mathrm{C}\left(3^{\prime}\right)\right)$; 1.62-1.50 (m, 2H, $\left.\mathrm{H}_{2} \mathrm{C}\left(4^{\prime}\right)\right)$; 1.40-1.34 (m, 1H, $\left.\mathrm{H}_{2} \mathrm{C}\left(3^{\prime}\right)\right) .{ }^{13} \mathrm{C}$ NMR $\left(\mathrm{CDCl}_{3}\right)$ : ' $139.4,135.6$ (2 C(arom.)); 136.9 (HC(2)); 130.9, 130.5, 
129.0, 128.9, 128.5, 128.1, 127.2 (7 signals for $10 \mathrm{HC}($ arom.) $+2 \mathrm{C}$ (imid.)); 63.7 $\left(\mathrm{C}\left(2^{\prime}\right)\right)$; $59.5\left(\mathrm{CH}_{2}-\mathrm{Ph}\right) ; 54.6\left(\mathrm{C}\left(5^{\prime}\right)\right) ; 49.8\left(\mathrm{CH}_{2}-\mathrm{N}\right)$; $29.1\left(\mathrm{C}\left(3^{\prime}\right)\right) ; 23.1\left(\mathrm{C}\left(4^{\prime}\right)\right) ; 13.4$ $\left(\mathrm{H}_{3} \mathrm{C}-\mathrm{C}(4)\right)$. HR-ESI-MS (MeOH + $\left.\mathrm{HCOOH}\right): 332.2121$ (calcd 332.2121 for $\mathrm{C}_{22} \mathrm{H}_{26} \mathrm{~N}_{3}$, $\left.[M+1]^{+}\right) \cdot[ \pm]_{\mathrm{D}}^{25}=-25\left(c=1.0, \mathrm{CH}_{2} \mathrm{Cl}_{2}\right)$.

\subsubsection{4,5-Diphenyl-1-\{[(2S)- $N$-benzylpyrrolidin-2-yl $]$ methyl $\}-1 H$-imidazole $6 \mathrm{c}$}

Yield: $1.3 \mathrm{~g}(68 \%)$. Colorless crystals. Mp $118-119{ }^{\circ} \mathrm{C}\left(\mathrm{CH}_{2} \mathrm{Cl}_{2} /\right.$ hexane). IR (KBr): v 3432br, 3085m, 2971m, 2927m, 2905m, 2786m, 2723w, 1603m, 1505m, 1452m, 1369m, 1256m, 774m, 698s, 653m. ${ }^{1} \mathrm{H}$ NMR $\left(\mathrm{CDCl}_{3}\right)$ : ' 7.76 (s, 1H, HC(2)); 7.54-7.45 (m, 5H, HC(arom.)); 7.39-7.36 (m, 2H, HC(arom.)); 7.33-7.28 (m, 2H, HC(arom.)); 7.27-7.21 (m, 5H, HC(arom.)); 7.17-7.14 (m, 1H, HC(arom.)); 3.88 (dd, $\left.{ }^{2} J_{\mathrm{H}, \mathrm{H}}=14.4,{ }^{3} J_{\mathrm{H}, \mathrm{H}}=5.4,1 \mathrm{H}, \mathrm{H}_{2} \mathrm{C}-\mathrm{N}\right) ; 3.74\left(\mathrm{dd},{ }^{2} J_{\mathrm{H}, \mathrm{H}}=14.4,{ }^{3} J_{\mathrm{H}, \mathrm{H}}=7.2,1 \mathrm{H}, \mathrm{H}_{2} \mathrm{C}-\mathrm{N}\right)$; 3.58, $3.25\left(\mathrm{AB}, J_{A B}=12.6,2 \mathrm{H}, \mathrm{H}_{2} \mathrm{C}-\mathrm{Ph}\right) ; 2.96-2.91\left(\mathrm{~m}, 1 \mathrm{H}, \mathrm{H}_{2} \mathrm{C}\left(5^{\prime}\right)\right)$; 2.72-2.66 (m, $\left.1 \mathrm{H}, \mathrm{HC}\left(2^{\prime}\right)\right)$; 2.26-2.19 (m, 1H, $\mathrm{H}_{2} \mathrm{C}\left(5^{\prime}\right)$ ); 1.83-1.76 (m, 1H, $\mathrm{H}_{2} \mathrm{C}\left(4^{\prime}\right)$ ); 1.70-1.58 (m, $\left.2 \mathrm{H}, \mathrm{H}_{2} \mathrm{C}\left(3^{\prime}\right)\right)$; 1.52-1.45 (m, 1H, $\left.\mathrm{H}_{2} \mathrm{C}\left(4^{\prime}\right)\right)$. ${ }^{13} \mathrm{C} \mathrm{NMR}\left(\mathrm{CDCl}_{3}\right)$ : ' $139.2,138.2,134.9$ (3 $\mathrm{C}(\operatorname{arom})) ;$.137.7 (HC(2)); 131.4, 131.3, 129.3, 129.0, 128.9, 128.7, 128.5, 128.3, 127.2, 126.8, 126.4 (15 CH(arom.), 2 C(imid.)); 63.7 (HC(2’')); $59.6\left(\mathrm{CH}_{2}-\mathrm{Ph}\right) ; 54.6$ $\left(\mathrm{H}_{2} \mathrm{C}\left(5^{\prime}\right)\right) ; 49.4\left(\mathrm{CH}_{2}-\mathrm{N}\right) ; 29.1\left(\mathrm{C}\left(4^{\prime}\right)\right) ; 23.2\left(\mathrm{C}\left(3^{\prime}\right)\right)$. HR-ESI-MS (MeOH + $\left.\mathrm{HCOOH}\right)$ : 394.2278 (calcd 394.2278 for $\left.\mathrm{C}_{27} \mathrm{H}_{28} \mathrm{~N}_{3},[M+1]^{+}\right)$. $[ \pm]_{\mathrm{D}}{ }^{25}=-31\left(c=1.0, \mathrm{CH}_{2} \mathrm{Cl}_{2}\right)$.

\subsubsection{4. $\quad N$-Phenyl-1- $\{[(2 S)-N$-benzylpyrrolidin-2-yl $]$ methyl $\}-5$-methyl-1H-}

\section{imidazole-4-carboxamide $6 \mathrm{~d}$}

Yield: $1.3 \mathrm{~g}(72 \%)$. Colorless crystals. $\mathrm{Mp} 82-84{ }^{\circ} \mathrm{C}\left(\mathrm{Et}_{2} \mathrm{O}\right)$. IR (KBr): v 3432br, $3270 \mathrm{~m}, 3243 \mathrm{~m}, 3121 \mathrm{~m}, 3033 \mathrm{~m}, 2969 \mathrm{~m}, 2946 \mathrm{~m}, 2779 \mathrm{~m}, 1943 \mathrm{w}, 1968 \mathrm{w}, 1752 \mathrm{w}$, 1660s, 1596s, 1537m, 1503s, 1440s, 1246s, 878m, 759s, 697s. ${ }^{1} \mathrm{H}$ NMR $\left(\mathrm{CDCl}_{3}\right)$ : 9.03 (s, 1H, NH); 7.70-7.67 (m, 2H, HC(arom.)); 7.48 (s, 1H, HC(2)); 7.35-7.28 (m, 6H, HC(arom.)); 7.27-7.24 (m, 1H, HC(arom.)); 7.09-7.07 (m, 1H, HC(arom.)); 3.81 $\left(\mathrm{dd},{ }^{2} J_{\mathrm{H}, \mathrm{H}}=14.4,{ }^{3} J_{\mathrm{H}, \mathrm{H}}=4.8,1 \mathrm{H}, \mathrm{H}_{2} \mathrm{C}-\mathrm{N}\right) ; 3.76\left(\mathrm{dd},{ }^{2} J_{\mathrm{H}, \mathrm{H}}=14.4,{ }^{3} J_{\mathrm{H}, \mathrm{H}}=6.0,1 \mathrm{H}, \mathrm{H}_{2} \mathrm{C}-\right.$ $\mathrm{N}) ; 3.75,3.49\left(\mathrm{AB}, J_{A B}=13.2,2 \mathrm{H}, \mathrm{H}_{2} \mathrm{C}-\mathrm{Ph}\right) ; 3.04-2.98\left(\mathrm{~m}, 1 \mathrm{H}, \mathrm{H}_{2} \mathrm{C}\left(5^{\prime}\right)\right) ; 2.96-2.90$ (m, 1H, $\left.\mathrm{HC}\left(2^{\prime}\right)\right) ; 2.57$ (s, 3H, $\left.\mathrm{H}_{3} \mathrm{C}-\mathrm{C}(5)\right)$; 2.39-2.31 (m, 1H, $\mathrm{H}_{2} \mathrm{C}\left(5^{\prime}\right)$ ); 1.93-1.85 (m, $1 \mathrm{H}, \mathrm{H}_{2} \mathrm{C}\left(3^{\prime}\right)$ ); 1.75-1.69 (m, 1H, $\mathrm{H}_{2} \mathrm{C}\left(4^{\prime}\right)$ ); 1.67-1.58 (m, 1H, $\mathrm{H}_{2} \mathrm{C}\left(4^{\prime}\right)$ ); 1.54-1.46 (m, $\left.1 \mathrm{H}, \mathrm{H}_{2} \mathrm{C}\left(3^{\prime}\right)\right)$. ${ }^{13} \mathrm{C} \mathrm{NMR}\left(\mathrm{CDCl}_{3}\right)$ : ' $162.1(\mathrm{C}=\mathrm{O}) ; 139.3,138.7$ (2 C(arom.)); 136.1 (HC(2)); 133.2, 131.5, 129.1, 128.9, 128.7, 127.5, 123.7, 119.7 (10 HC(arom.), 2 C(imid.)); 63.4 (HC(2')); $60.2\left(\mathrm{CH}_{2}-\mathrm{Ph}\right) ; 55.0\left(\mathrm{H}_{2} \mathrm{C}\left(5^{\prime}\right)\right) ; 49.0\left(\mathrm{CH}_{2}-\mathrm{N}\right) ; 29.0\left(\mathrm{C}\left(3^{\prime}\right)\right)$; 
$23.4\left(\mathrm{C}\left(4^{\prime}\right)\right)$; $9.9\left(\mathrm{H}_{3} \mathrm{C}-\mathrm{C}(5)\right)$. HR-ESI-MS (MeOH + $\left.\mathrm{HCOOH}\right): 375.2178$ (calcd 375.2179 for $\left.\mathrm{C}_{23} \mathrm{H}_{27} \mathrm{~N}_{4} \mathrm{O},[M+1]^{+}\right)$. $[ \pm]_{\mathrm{D}}{ }^{25}=-11\left(c=1.0, \mathrm{CH}_{2} \mathrm{Cl}_{2}\right)$.

\subsubsection{5. $\quad N$-(4-Bromophenyl)-1- $\{[(2 S)-N$-benzylpyrrolidin-2-yl]methyl $\}-5$-methyl-} $1 H$-imidazole-4-carboxamide $6 \mathrm{e}$

Yield: $2.3 \mathrm{~g}(63 \%)$. Colorless oil $\left(\mathrm{SiO}_{2}, \mathrm{MeOH} / \mathrm{AcOEt}, 1: 3\right)$. IR (film): $v$ 3362br, 3180s, 3061m, 3028m, 2970m, 2872m, 2799m, 1951m, 1888m, 1671m, 1588m, $1506 \mathrm{~m}, 1397 \mathrm{~m}, 1241 \mathrm{~m}, 1116 \mathrm{~m}, 1060 \mathrm{~m}, 1007 \mathrm{~s}, 829 \mathrm{~s}, 700 \mathrm{~m}, 659 \mathrm{~m} .{ }^{1} \mathrm{H}$ NMR $\left(\mathrm{CDCl}_{3}\right)$ : ' 9.03 (s, 1H, NH); 7.69-7.57 (m, 2H, HC(arom.)); 7.48 (s, 1H, HC(2)); 7.44-7.41 (m, 1H, HC(arom.)); 7.34-7.24 (m, 6H, HC(arom.)); 3.80-3.74 (m, 2H, $\left.\mathrm{H}_{2} \mathrm{C}-\mathrm{N}\right) ; 3.73,3.50$ $\left(\mathrm{AB}, J_{A B}=13.2,2 \mathrm{H}, \mathrm{H}_{2} \mathrm{C}-\mathrm{Ph}\right) ; 3.04-2.98\left(\mathrm{~m}, 1 \mathrm{H}, \mathrm{H}_{2} \mathrm{C}\left(5^{\prime}\right)\right) ; 2.96-2.91(\mathrm{~m}, 1 \mathrm{H}$, $\left.\mathrm{HC}\left(2^{\prime}\right)\right) ; 2.55$ (s, 3H, $\left.\mathrm{H}_{3} \mathrm{C}-\mathrm{C}(5)\right)$; 2.38-2.31 (m, 1H, $\left.\mathrm{H}_{2} \mathrm{C}\left(5^{\prime}\right)\right)$; 1.94-1.86 (m, 1H, $\left.\mathrm{H}_{2} \mathrm{C}\left(4^{\prime}\right)\right)$; 1.76-1.69 (m, 1H, $\left.\mathrm{H}_{2} \mathrm{C}\left(3^{\prime}\right)\right)$; 1.66-1.58 (m, 1H, $\left.\mathrm{H}_{2} \mathrm{C}\left(3^{\prime}\right)\right)$; 1.53-1.46 (m, 1H, $\left.\mathrm{H}_{2} \mathrm{C}\left(4^{\prime}\right)\right) .{ }^{13} \mathrm{C}$ NMR $\left(\mathrm{CDCl}_{3}\right)$ : ' $162.0(\mathrm{C}=\mathrm{O}) ; 139.3,137.8$ (2 $\mathrm{C}($ arom. $\left.)\right) ; 136.3$ (HC(2)); 132.1, 129.1, 129.0, 128.7, 127.5, 121.2, 119.7, 116.1 (1 C(arom.), 9 CH(arom.), 2 C(imid.)); 63.4 (HC(2')); $60.2\left(\mathrm{CH}_{2}-\mathrm{Ph}\right) ; 55.0\left(\mathrm{H}_{2} \mathrm{C}\left(5^{\prime}\right)\right) ; 49.0\left(\mathrm{CH}_{2}-\mathrm{N}\right)$; 29.0 (C(3')); 23.4 (C(4’)); 9.9 ( $\left.\mathrm{H}_{3} \mathrm{C}-\mathrm{C}(5)\right)$. HR-ESI-MS (MeOH + $\left.\mathrm{HCOOH}\right): 453.1279$ (calcd 453.1285 for $\left.\mathrm{C}_{23} \mathrm{H}_{26} \mathrm{BrN}_{4} \mathrm{O},\left[M\left({ }^{79} \mathrm{Br}\right)+1\right]^{+}\right)$. $[ \pm]_{\mathrm{D}}{ }^{25}=-10\left(c=1.0, \mathrm{CH}_{2} \mathrm{Cl}_{2}\right)$.

\subsubsection{General procedure for synthesis of imidazoles $7 \mathrm{a}$ and $7 \mathrm{~d}$}

To a magnetically stirred solution of an imidazole $N$-oxide 5 ( $1 \mathrm{mmol})$ in $\mathrm{MeOH}(4 \mathrm{ml})$ $10 \% \mathrm{Pd} / \mathrm{C}(0.05 \mathrm{~g})$ was added and the mixture stirred under $\mathrm{H}_{2}$ atm until the reaction was completed (monitored by TLC). Then, the solid was filtered trough celite and the solvent was evaporated under reduced pressure. The obtained product was purified by column chromatography or by crystallization.

\subsubsection{4,5-Diphenyl-1-[(2S)-pyrrolidin-2-ylmethyl]-1H-imidazole 7a}

Yield: $0.277 \mathrm{mg}(87 \%)$. Colorless crystals. $\mathrm{Mp} 123-125{ }^{\circ} \mathrm{C}\left(\mathrm{CH}_{2} \mathrm{Cl}_{2} /\right.$ hexane). IR (KBr): v 3357br, 3049m, 2989m, 2834m, 2223w, 1509m, 1439m, 1357m, 1220m, 1033m, 907m, 895m, 802m, 731m. ${ }^{1} \mathrm{H}$ NMR $\left(\mathrm{CDCl}_{3}\right)$ : ' 7.74 (s, 1H, NH); 7.48-7.43 (m, 5H, HC(arom.)); 7.35-7.32 (m, 2H, HC(arom.)); 7.21-7.16 (m, 2H, HC(arom.)); $7.16-7.10\left(\mathrm{~m}, 1 \mathrm{H}, \mathrm{HC}\right.$ (arom.)); $3.77\left(\mathrm{dd},{ }^{2} J_{\mathrm{H}, \mathrm{H}}=12.0,{ }^{3} J_{\mathrm{H}, \mathrm{H}}=6.0,1 \mathrm{H}, \mathrm{H}_{2} \mathrm{C}-\mathrm{N}\right) ; 3.74$ $\left(\mathrm{dd},{ }^{2} J_{\mathrm{H}, \mathrm{H}}=12.0,{ }^{3} J_{\mathrm{H}, \mathrm{H}}=3.6,1 \mathrm{H}, \mathrm{H}_{2} \mathrm{C}-\mathrm{N}\right) ; 3.24-3.17\left(\mathrm{~m}, 1 \mathrm{H}, \mathrm{HC}\left(2^{\prime}\right)\right) ; 2.92-2.84(\mathrm{~m}$, 
$\left.2 \mathrm{H}, \mathrm{H}_{2} \mathrm{C}\left(5^{\prime}\right)\right)$; 1.78-1.61 (m, $\left.1 \mathrm{H}, \mathrm{H}_{2} \mathrm{C}\left(3^{\prime}\right), 2 \mathrm{H}, \mathrm{H}_{2} \mathrm{C}\left(4^{\prime}\right)\right)$; 1.32-1.24 (m, $1 \mathrm{H}, \mathrm{H}_{2} \mathrm{C}\left(3^{\prime}\right)$. ${ }^{13} \mathrm{C}$ NMR $\left(\mathrm{CDCl}_{3}\right)$ : ' 138.4, 137.4, 134.9, 131.3, 131.2, 129.3, 128.9, 128.6, 128.3, 126.8, 126.4 (10 HC(arom.), 2 (C(arom.), 3 C(imid.)); 58.6 (HC(2')); $50.5\left(\mathrm{CH}_{2}-\mathrm{N}\right)$; $46.4\left(\mathrm{H}_{2} \mathrm{C}\left(5^{\prime}\right)\right) ; 29.4\left(\mathrm{C}\left(4^{\prime}\right)\right) ; 25.3\left(\mathrm{C}\left(3^{\prime}\right)\right)$. HR-ESI-MS (MeOH + $\left.\mathrm{HCOOH}\right): 302.1687$ (calcd 302.1685 for $\left.\mathrm{C}_{17} \mathrm{H}_{24} \mathrm{~N}_{3} \mathrm{~S},[M+1]^{+}\right)$. $[ \pm]_{\mathrm{D}}{ }^{25}=-18\left(c=1.0, \mathrm{CH}_{2} \mathrm{Cl}_{2}\right)$.

\subsubsection{5-Methyl- $N$-phenyl-1-[(2S)-pyrrolidin-2-ylmethyl]-1H-imidazole-4- carboxamide $7 b$}

Yield: $0.235 \mathrm{mg}$ (83\%). Colorless oil $\left(\mathrm{SiO}_{2}, \mathrm{MeOH} / \mathrm{AcOEt}, 6: 4\right)$. IR (film): $v$ 3366br, 3058m, 2961m, 2873m, 2246w, 1663m, 1597m, 1575m, 1442m, 1330m, 1243m, 1064m, 906m, 877m, 829m, 756m. ${ }^{1} \mathrm{H} \mathrm{NMR}\left(\mathrm{CDCl}_{3}\right)$ : ' 9.05 (s, 1H, NH); 7.68-7.64 (m, 2H, HC(arom.)); 7.47 (s, 1H, HC(2)); 7.33-7.28 (m, 2H, HC(arom.)); $7.08-7.03(\mathrm{~m}, 1 \mathrm{H}, \mathrm{HC}(\operatorname{arom}).) ; 3.86\left(\mathrm{dd},{ }^{2} J_{\mathrm{H}, \mathrm{H}}=14.1,{ }^{3} J_{\mathrm{H}, \mathrm{H}}=5.4,1 \mathrm{H}, \mathrm{H}_{2} \mathrm{C}-\mathrm{N}\right) ; 3.78$ $\left(\mathrm{dd},{ }^{2} J_{\mathrm{H}, \mathrm{H}}=14.1,{ }^{3} J_{\mathrm{H}, \mathrm{H}}=7.8,1 \mathrm{H}, \mathrm{H}_{2} \mathrm{C}-\mathrm{N}\right) ; 3.41-3.35\left(\mathrm{~m}, 1 \mathrm{H}, \mathrm{HC}\left(2^{\prime}\right)\right)$; 2.94-2.89 (m, $2 \mathrm{H}, \mathrm{H}_{2} \mathrm{C}\left(5^{\prime}\right)$ ); 2.60 (s, 3H, $\left.\mathrm{H}_{3} \mathrm{C}-\mathrm{N}\right)$ ) 1.93-1.86 (m, 1H, $\mathrm{H}_{2} \mathrm{C}\left(3^{\prime}\right)$ ); 1.84-1.68 (m, 2H, $\left.\mathrm{H}_{2} \mathrm{C}\left(4^{\prime}\right)\right)$; $1.44-1.37\left(\mathrm{~m}, 1 \mathrm{H}, \mathrm{H}_{2} \mathrm{C}\left(3^{\prime}\right)\right) .{ }^{13} \mathrm{C} \mathrm{NMR}\left(\mathrm{CDCl}_{3}\right)$ : ' $162.1(\mathrm{C}=\mathrm{O}) ; 138.6$ (1 C(arom.)); 135.9, 133.1, 131.5, 129.0, 123.7, 119.7 (5 HC(arom.), 3 C(imid.)); 58.2 $\left(\mathrm{CH}_{2}-\mathrm{N}\right)$; $50.1\left(\mathrm{HC}\left(2^{\prime}\right)\right)$; $46.5\left(\mathrm{H}_{2} \mathrm{C}\left(5^{\prime}\right)\right)$; $29.4\left(\mathrm{C}\left(3^{\prime}\right)\right)$; $25.4\left(\mathrm{C}\left(4^{\prime}\right)\right)$; $9.8\left(\mathrm{CH}_{3}-\mathrm{N}\right)$. HRESI-MS $(\mathrm{MeOH}+\mathrm{HCOOH}): 302.1687$ (calcd 302.1685 for $\mathrm{C}_{17} \mathrm{H}_{24} \mathrm{~N}_{3} \mathrm{~S},[M+1]^{+}$). $[ \pm]_{\mathrm{D}}^{25}=-24\left(c=1.0, \mathrm{CH}_{2} \mathrm{Cl}_{2}\right)$.

\subsubsection{Synthesis of imidazole-2-thiones 9}

To a magnetically stirred solution of an imidazole $\mathrm{N}$-oxide 5 (4 mmol) in $\mathrm{CHCl}_{3}(4 \mathrm{ml})$, 2,2,4,4-tetramethylcyclobutane-1,3-dithione 8 (2 mmol) in $\mathrm{CHCl}_{3}(2 \mathrm{ml})$ was added dropwise at $0{ }^{\circ} \mathrm{C}$, and stirring was continued overnight at rt. Then, the solvent was evaporated under reduced pressure and the residue was washed with hexane. The resulting material was purified by crystallization.

\subsubsection{1- $\{[(2 S)-N$-Benzylpyrrolidin-2-yl]methyl $\}-4,5$-dimethyl-1,3-dihydro- $2 H$ - imidazole-2-thione 9a}

Yield: $0.8 \mathrm{~g}(68 \%)$. Colorless crystals. Mp $108-110{ }^{\circ} \mathrm{C}\left(\mathrm{CH}_{2} \mathrm{Cl}_{2} /\right.$ hexane). IR $(\mathrm{KBr}): v$ 3432br, 3085m, 2943m, 2792m, 2522w, 1659s, 1499s, 1452m, 1393m, 1306m, 1127m, 1029m, 782m, 739s, 697s, 472s. ${ }^{1} \mathrm{H}$ NMR $\left(\mathrm{CDCl}_{3}\right)$ : $^{\prime} 11.45$ (s, $1 \mathrm{H}$, 
$\mathrm{NH}) ; 7.27-7.23$ (m, 4H, HC(arom.)); 7.21-7.17 (m, 1H, HC(arom.)); $4.02\left(\mathrm{dd},{ }^{2} J_{\mathrm{H}, \mathrm{H}}=\right.$ $\left.13.3,{ }^{3} J_{\mathrm{H}, \mathrm{H}}=7.8,1 \mathrm{H}, \mathrm{H}_{2} \mathrm{C}-\mathrm{N}\right) ; 3.78\left(\mathrm{dd},{ }^{2} J_{\mathrm{H}, \mathrm{H}}=13.3,{ }^{3} J_{\mathrm{H}, \mathrm{H}}=6.0,1 \mathrm{H}, \mathrm{H}_{2} \mathrm{C}-\mathrm{N}\right) ; 3.87$, $3.45\left(\mathrm{AB}, J_{A B}=13.2,2 \mathrm{H}, \mathrm{H}_{2} \mathrm{C}-\mathrm{Ph}\right) ; 3.41-3.34\left(\mathrm{~m}, 1 \mathrm{H}, \mathrm{HC}\left(2^{\prime}\right)\right)$; 2.99-2.94 (m, 1H, $\left.\mathrm{H}_{2} \mathrm{C}\left(5^{\prime}\right)\right) ; 2.28$ (m, 1H, $\left.\mathrm{H}_{2} \mathrm{C}\left(5^{\prime}\right)\right) ; 2.03$ (s, 3H, $\left.\mathrm{H}_{3} \mathrm{C}-\mathrm{C}(4)\right) ; 2.01$ (s, 3H, $\left.\mathrm{H}_{3} \mathrm{C}-\mathrm{C}(5)\right)$; 1.88-1.63 (m, 2H, $\left.\mathrm{H}_{2} \mathrm{C}\left(4^{\prime}\right), 2 \mathrm{H}, \mathrm{H}_{2} \mathrm{C}\left(3^{\prime}\right)\right) .{ }^{13} \mathrm{C} \mathrm{NMR}\left(\mathrm{CDCl}_{3}\right)$ ) ' $158.5(\mathrm{C}=\mathrm{S}) ; 139.2$ (1 C(arom.)); 129.0, 128.3, 127.0, 122.2, 119.9 (5 HC(arom.), 1 (C(arom.), 2 C(imid.)); $61.9\left(\mathrm{HC}\left(2^{\prime}\right)\right) ; 60.5\left(\mathrm{CH}_{2}-\mathrm{Ph}\right) ; 54.8\left(\mathrm{H}_{2} \mathrm{C}\left(5^{\prime}\right)\right) ; 49.4\left(\mathrm{CH}_{2}-\mathrm{N}\right) ; 29.2\left(\mathrm{C}\left(4^{\prime}\right)\right) ; 23.6$ $\left(\mathrm{C}\left(3^{\prime}\right)\right)$; $9.3\left(\mathrm{H}_{3} \mathrm{C}-\mathrm{C}(5)\right)$; $9.2\left(\mathrm{H}_{3} \mathrm{C}-\mathrm{C}(4)\right)$. HR-ESI-MS (MeOH + $\left.\mathrm{HCOOH}\right): 302.1687$ (calcd 302.1685 for $\left.\mathrm{C}_{17} \mathrm{H}_{24} \mathrm{~N}_{3} \mathrm{~S},[M+1]^{+}\right)$. [ $]_{\mathrm{D}}{ }^{25}=-19\left(c=1.0, \mathrm{CH}_{2} \mathrm{Cl}_{2}\right)$.

\subsubsection{1- $\{[(2 S)-N$-Benzylpyrrolidin-2-yl]methyl $\}-4,5-d i p h e n y l-1,3-d i h y d r o-2 H$ - imidazole-2-thione 9b}

Yield: 0.5 g (31\%). Colorless crystals. Mp 197-199 ${ }^{\circ} \mathrm{C}(\mathrm{EtOH}) . \mathrm{IR}(\mathrm{KBr}): v$ $3432 \mathrm{br}, 3058 \mathrm{~m}, 2937 \mathrm{~m}, 2807 \mathrm{~m}, 1602 \mathrm{~m}, 1492 \mathrm{~s}, 1397 \mathrm{~s}, 1202 \mathrm{~m}, 1160 \mathrm{~m}, 1119 \mathrm{~m}, 1073 \mathrm{~m}$, 1027m, 700s, 682m, 556s. ${ }^{1} \mathrm{H}$ NMR $\left(\mathrm{CDCl}_{3}\right)$ : ‘ 11.30 (s, 1H, NH); 7.46-7.40 (m, 3H, HC(arom.)); 7.36-7.33 (m, 2H, HC(arom.)); 7.25-7.16 (m, 10H, HC(arom.)); 4.12 (dd, $\left.{ }^{2} J_{\mathrm{H}, \mathrm{H}}=12.9,{ }^{3} J_{\mathrm{H}, \mathrm{H}}=9.0,1 \mathrm{H}, \mathrm{H}_{2} \mathrm{C}-\mathrm{N}\right) ; 3.94\left(\mathrm{dd},{ }^{2} J_{\mathrm{H}, \mathrm{H}}=12.9,{ }^{3} J_{\mathrm{H}, \mathrm{H}}=4.2,1 \mathrm{H}, \mathrm{H}_{2} \mathrm{C}-\mathrm{N}\right)$; 3.76, $3.22\left(\mathrm{AB}, J_{A B}=12.9,2 \mathrm{H}, \mathrm{H}_{2} \mathrm{C}-\mathrm{Ph}\right) ; 3.21-3.25$ (m, 1H, $\left.\mathrm{HC}\left(2^{\prime}\right)\right)$; 2.81-2.76 (m, $\left.1 \mathrm{H}, \mathrm{H}_{2} \mathrm{C}\left(5^{\prime}\right)\right)$; 2.20-2.15 (m, 1H, $\left.\mathrm{H}_{2} \mathrm{C}\left(5^{\prime}\right)\right)$; 1.73-1.66 (m, $\left.1 \mathrm{H}, \mathrm{H}_{2} \mathrm{C}\left(4^{\prime}\right)\right) .{ }^{13} \mathrm{C} \mathrm{NMR}$ $\left(\mathrm{CDCl}_{3}\right)$ : ' $161.4(\mathrm{C}=\mathrm{S})$; 140.1 (1 C(arom.)); 131.7, 129.6, 129.3, 129.1, 129.0, 128.9, 128.3, 128.1, 127.9, 126.9, 126.8, 125.5 (12 signals for $15 \mathrm{HC}$ (arom.), $3 \mathrm{C}$ (arom.), 2 C(imid.)); 61.7 (HC(2')); $59.9\left(\mathrm{CH}_{2}-\mathrm{Ph}\right) ; 54.3\left(\mathrm{C}\left(5^{\prime}\right)\right) ; 49.3\left(\mathrm{CH}_{2}-\mathrm{N}\right) ; 29.1$ (C(3’)); $23.5\left(\mathrm{C}\left(4^{\prime}\right)\right)$. HR-ESI-MS (MeOH + HCOOH): 426.1998 (calcd 426.1999 for $\left.\mathrm{C}_{27} \mathrm{H}_{28} \mathrm{~N}_{3} \mathrm{~S},[M+1]^{+}\right) .[ \pm]_{\mathrm{D}}^{25}=-14\left(c=1.0, \mathrm{CH}_{2} \mathrm{Cl}_{2}\right)$.

\subsubsection{3. $N$-(4-Bromophenyl)-1- $\{[(2 S)-N$-benzylpyrrolidin-2-yl] methyl $\}-5$-methyl-2- thioxo-2,3-dihydro-1H-imidazole-4-carboxamide 9c}

Yield: 0.8 g (41\%). Colorless crystals. Mp $215{ }^{\circ} \mathrm{C}$ (EtOH) (decomposition). IR (KBr): v 3432br, 3275m, 3125m, 3065m, 2952m, 2902w, 2787m, 1663s, 1625s, 1542s, 1489s, 1380m, 828m, 811m, 698m, 504m. ${ }^{1} \mathrm{H} \mathrm{NMR}\left(\mathrm{CDCl}_{3}\right)$ : ' 8.85 (s, 1H, HN); 7.73-7.66 (m, 2H, HC(arom.)); 7.45-7.41 (m, 2H, HC(arom.)); 7.24-7.20 (m, 4H, $\mathrm{HC}$ (arom.)); 7.15-7.10 (m, 1H, HC(arom.)); 4.11 (dd, $J_{\mathrm{H}, \mathrm{H}}=13.3, J_{\mathrm{H}, \mathrm{H}}=5.8,1 \mathrm{H}, \mathrm{H}_{2} \mathrm{C}-$ $\mathrm{N}) ; 3.85\left(\mathrm{dd}, J_{\mathrm{H}, \mathrm{H}}=13.3, J_{\mathrm{H}, \mathrm{H}}=6.0,1 \mathrm{H}, \mathrm{H}_{2} \mathrm{C}-\mathrm{N}\right) ; 3.74,3.19\left(\mathrm{AB}, J_{A B}=12.9,2 \mathrm{H}\right.$, $\left.\mathrm{H}_{2} \mathrm{C}-\mathrm{Ph}\right) ; 3.42-3.33\left(\mathrm{~m}, 1 \mathrm{H}, \mathrm{H}_{2} \mathrm{C}\left(5^{\prime}\right)\right)$; 3.08-3.02 (m, 1H, $\left.\mathrm{HC}\left(2^{\prime}\right)\right)$; 2.57 (s, 3H, $\mathrm{H}_{3} \mathrm{C}-$ 
$\mathrm{C}(5)) ; 2.46-2.35\left(\mathrm{~m}, 1 \mathrm{H}, \mathrm{H}_{2} \mathrm{C}\left(5^{\prime}\right)\right)$; 1.92-1.85 (m, 1H, $\left.\mathrm{H}_{2} \mathrm{C}\left(4^{\prime}\right)\right)$; 1.83-1.68 (m, 2H, $\left.\mathrm{H}_{2} \mathrm{C}\left(3^{\prime}\right), 1 \mathrm{H} \mathrm{H}_{2} \mathrm{C}\left(4^{\prime}\right)\right) .{ }^{13} \mathrm{C} \mathrm{NMR}\left(\mathrm{CDCl}_{3}\right)$ : ' $162.1(\mathrm{C}=\mathrm{S}) ; 161.6(\mathrm{C}=\mathrm{O}) ; 138.7,138.0(2$ $\mathrm{C}$ (arom.)); 136.6 (HC(2)); 132.7, 129.6, 129.1, 128.9, 121.7, 119.7, 116.1 (9 HC(arom.), 1 C(arom.)), 2 C(imid.)); 63.8 (C(2')); $60.9\left(\mathrm{CH}_{2}-\mathrm{Ph}\right) ; 55.3$ (C(5')); 49.2 $\left(\mathrm{CH}_{2}-\mathrm{N}\right) ; 29.7\left(\mathrm{C}\left(3^{\prime}\right)\right) ; 23.1\left(\mathrm{C}\left(4^{\prime}\right)\right) ; 10.0\left(\mathrm{H}_{3} \mathrm{C}-\mathrm{C}(5)\right)$. HR-ESI-MS (MeOH + $\mathrm{HCOOH}): 485.1002\left(\right.$ calcd 485.1005 for $\left.\mathrm{C}_{23} \mathrm{H}_{26} \mathrm{BrN}_{4} \mathrm{OS},[M+1]^{+}\right) .[ \pm]_{\mathrm{D}}^{25}=-10(\mathrm{c}=$ $1.0, \mathrm{CH}_{2} \mathrm{Cl}_{2}$ ).

\subsubsection{Synthesis of 3-alkylimidazolium tetrafluoroborates 11}

A solution of an imidazole $6(5 \mathrm{mmol})$ and an alkyl bromide $(5 \mathrm{mmol})$ in acetonitrile $(5 \mathrm{ml})$ was heated at reflux for $16 \mathrm{~h}$. Then, the solvent was removed under reduced pressure, the resulting product was washed with $\mathrm{Et}_{2} \mathrm{O}(3 \times 5 \mathrm{ml})$, and dried under high vacuum for $4 \mathrm{~h}$. The imidazolium bromide obtained was magnetically stirred with an equimolar amount of solid $\mathrm{NaBF}_{4}$ in acetone $(5 \mathrm{ml})$ overnight at $\mathrm{rt}$. The resulting suspension was filtered through neutral alumina $\left(\mathrm{CHCl}_{3}\right)$ and the solvent evaporated under reduced pressure.

4.2.8.1. $\quad 1-\{[(2 S)-N$-Benzylpyrrolidin-2-yl]methyl $\}-3-h e x y l-4,5-d i m e t h y l-1 H$ imidazolium tetrafluoroborate 11a

Yield: $1.6 \mathrm{~g}(75 \%)$. Pale yellow oil $\left(\mathrm{Al}_{2} \mathrm{O}_{3}, \mathrm{CHCl}_{3}\right)$. IR (film): v 3648w, 3628w, 3566m, 3154m, 3087m, 2956m, 2931m, 2805m, 1818m, 1564m, 1455m, 1058br, 742m, 703m, 626m. ${ }^{1} \mathrm{H}$ NMR ( $\left.\mathrm{CDCl}_{3}\right)$ : ‘ 8.72 (HC(2)); 7.29-7.26 (m, 2H, HC(arom.)); 7.23-7.19 (m, 3H, HC(arom.)); 4.01-3.95 (m, 1H, $\mathrm{H}_{2} \mathrm{C}-\mathrm{N}, 2 \mathrm{H}, \mathrm{H}_{2} \mathrm{C}($ alk.)); 3.89 (dd, $\left.{ }^{2} J_{\mathrm{H}, \mathrm{H}}=13.8,{ }^{3} J_{\mathrm{H}, \mathrm{H}}=6.6,1 \mathrm{H}, \mathrm{H}_{2} \mathrm{C}-\mathrm{N}\right) ; 3.59,3.56\left(\mathrm{AB}, J_{A B}=13.2,2 \mathrm{H}, \mathrm{H}_{2} \mathrm{C}-\mathrm{Ph}\right) ; 3.16-$ 3.11 (m, 1H, $\left.\mathrm{HC}\left(2^{\prime}\right)\right)$; 3.10-3.06 (m, 1H, $\left.\mathrm{H}_{2} \mathrm{C}\left(5^{\prime}\right)\right)$; 2.50-2.45 (m, 1H, $\mathrm{H}_{2} \mathrm{C}\left(5^{\prime}\right)$ ); 2.16 (s, 3H, $\left.\mathrm{H}_{3} \mathrm{C}-\mathrm{C}(4)\right) ; 2.12$ (s, 3H, $\left.\mathrm{H}_{3} \mathrm{C}-\mathrm{C}(5)\right)$; 2.05-1.98 (m, 1H, $\mathrm{H}_{2} \mathrm{C}\left(3^{\prime}\right)$ ); 1.83-1.77 (m, $1 \mathrm{H}, \mathrm{H}_{2} \mathrm{C}\left(4^{\prime}\right)$ ); 1.74-1.67 (m, 1H, $\mathrm{H}_{2} \mathrm{C}\left(4^{\prime}\right)$; $2 \mathrm{H}, \mathrm{H}_{2} \mathrm{C}\left(\right.$ alk. )) $1.55-1.50$ (m, 1H, $\mathrm{H}_{2} \mathrm{C}\left(3^{\prime}\right)$ ); 1.32-1.24 (m, 6H, $\mathrm{H}_{2} \mathrm{C}\left(\right.$ alk.)); 0.85 (t, $J_{\mathrm{H}, \mathrm{H}}=7.2,3 \mathrm{H}, \mathrm{H}_{3} \mathrm{C}\left(\right.$ alk. )). ${ }^{13} \mathrm{C} \mathrm{NMR}\left(\mathrm{CDCl}_{3}\right)$ : 139.4 (1 C(arom.); 135.4 (HC(2)); 128.9, 128.5, 127.3, 127.2, 126.0 (5 CH(arom.), 2 C(imid.)); 62.7 (HC(2')); $60.5\left(\mathrm{CH}_{2}-\mathrm{Ph}\right) ; 54.9\left(\mathrm{H}_{2} \mathrm{C}\left(5^{\prime}\right)\right) ; 50.8\left(\mathrm{CH}_{2}-\mathrm{N}\right) ; 47.4,31.3$, 29.9 (3 C(alk.)); 28.5 (C(4')); 26.1 (C(alk.)); 23.9 (C(3’)); 22.6 (C(alk.)); 14.1 $\left(\mathrm{H}_{3} \mathrm{C}(\right.$ alk. $\left.)\right) ; 8.7\left(\mathrm{H}_{3} \mathrm{C}-\mathrm{C}(5)\right) ; 8.5\left(\mathrm{H}_{3} \mathrm{C}-\mathrm{C}(4)\right) .[ \pm]_{\mathrm{D}}{ }^{25}=-35\left(c=1.0, \mathrm{CH}_{2} \mathrm{Cl}_{2}\right)$. 
4.2.8.2. $1-\{[(2 S)-N$-Benzylopyrrolidin-2-yl]methyl $\}-4,5$-dimethyl-3-octyl- $1 H$ imidazolium tetrafluoroborate $11 \mathrm{~b}$

Yield: $1.6 \mathrm{~g}(70 \%)$. Pale yellow oil $\left(\mathrm{Al}_{2} \mathrm{O}_{3}, \mathrm{CHCl}_{3}\right)$. IR (film): v 3584w, 3154m, $3087 \mathrm{~m}, 2928 \mathrm{~m}, 2857 \mathrm{~m}, 2806 \mathrm{~m}, 1733 \mathrm{w}, 1699 \mathrm{w}, 1565 \mathrm{~s}, 1454 \mathrm{~m}, 1202 \mathrm{~m}, 1058 \mathrm{br}, 828 \mathrm{w}$, 742m, 703m. ${ }^{1} \mathrm{H}$ NMR $\left(\mathrm{CDCl}_{3}\right)$ : ‘ 8.73 (s, 1H, $\left.\mathrm{HC}(2)\right)$; 7.28-7.25 (m, 2H, HC(arom.)); $7.23-7.18(\mathrm{~m}, 3 \mathrm{H}, \mathrm{HC}($ arom. $)) ; 3.98\left(\mathrm{dd},{ }^{2} J_{\mathrm{H}, \mathrm{H}}=14.4,{ }^{3} J_{\mathrm{H}, \mathrm{H}}=5.4,1 \mathrm{H}, \mathrm{H}_{2} \mathrm{C}-\mathrm{N}\right) ; 3.97-$ $3.94\left(\mathrm{~m}, 2 \mathrm{H}, \mathrm{H}_{2} \mathrm{C}(\right.$ alk. $\left.)\right) ; 3.88\left(\mathrm{dd},{ }^{2} J_{\mathrm{H}, \mathrm{H}}=14.4,{ }^{3} J_{\mathrm{H}, \mathrm{H}}=7.2,1 \mathrm{H}, \mathrm{H}_{2} \mathrm{C}-\mathrm{N}\right) ; 3.57,3.55$ $\left(\mathrm{AB}, J_{A B}=13.6,2 \mathrm{H}, \mathrm{H}_{2} \mathrm{C}-\mathrm{Ph}\right) ; 3.16-3.11\left(\mathrm{~m}, 1 \mathrm{H}, \mathrm{H}_{2} \mathrm{C}\left(2^{\prime}\right)\right) ; 3.10-3.05(\mathrm{~m}, 1 \mathrm{H}$, $\left.\mathrm{H}_{2} \mathrm{C}\left(5^{\prime}\right)\right)$; 2.50-2.44 (m, 1H, $\left.\mathrm{H}_{2} \mathrm{C}\left(5^{\prime}\right)\right)$; 2.15 (s, 3H, $\left.\mathrm{H}_{3} \mathrm{C}-\mathrm{C}(4)\right)$; 2.10 (s, 3H, $\mathrm{H}_{3} \mathrm{C}-$ $\mathrm{C}(5))$; 1.97-1.82 (m, 1H, $\left.\mathrm{H}_{2} \mathrm{C}\left(3^{\prime}\right)\right)$; 1.82-1.77 (m, 1H, $\left.\mathrm{H}_{2} \mathrm{C}\left(3^{\prime}\right)\right)$; 1.74-1.67 (m, 1H, $\mathrm{H}_{2} \mathrm{C}\left(4^{\prime}\right), 2 \mathrm{H}, \mathrm{H}_{2} \mathrm{C}\left(\right.$ alk.)); 1.55-1.49 (m, 1H, $\left.\mathrm{H}_{2} \mathrm{C}\left(4^{\prime}\right)\right)$; 1.31-1.21 (m, 10H, $\mathrm{H}_{2} \mathrm{C}($ alk.)); $8.62\left(\mathrm{t}, J_{\mathrm{H}, \mathrm{H}}=6.9, \mathrm{H}_{3} \mathrm{C}(\right.$ alk. $\left.)\right) .{ }^{13} \mathrm{C} \mathrm{NMR}\left(\mathrm{CDCl}_{3}\right)$ : ' 139.3 (1 C(arom.); $135.4(\mathrm{HC}(2))$; 128.9, 128.5, 127.2, 127.2, 126.0 (5 HC(arom.), 2 C(imid.)); 62.6 (HC(2')); $60.4\left(\mathrm{CH}_{2}-\right.$ $\mathrm{Ph}) ; 54.9$ (C(5')); $50.8\left(\mathrm{CH}_{2}-\mathrm{N}\right)$; 47.4, 31.8, 29.9, 29.2, 29.1 (5 C(alk.)); 28.4 (C(3')); 26.5 (C(alk.)); 23.9 (C(4')); 22.7 (C(alk.)); 14.2 ( $\mathrm{H}_{3} \mathrm{C}\left(\right.$ alk.)); $8.7\left(\mathrm{H}_{3} \mathrm{C}-\mathrm{C}(4)\right) ; 8.5$ $\left(\mathrm{H}_{3} \mathrm{C}-\mathrm{C}(5)\right) \cdot[ \pm]_{\mathrm{D}}{ }^{25}=-22\left(c=1.0, \mathrm{CH}_{2} \mathrm{Cl}_{2}\right)$.

\subsubsection{3. $1-\{[(2 S)-N$-Benzylpyrrolidin-2-yl $]$ methyl $\}-3-$ butyl-4-methyl-5-phenyl-1H- imidazolium tetrafluoroborate 11c}

Yield: $1.6 \mathrm{~g}(68 \%)$. Pale yellow oil $\left(\mathrm{Al}_{2} \mathrm{O}_{3}, \mathrm{CHCl}_{3}\right)$. IR (film): $v 3151 \mathrm{~m}, 3084 \mathrm{~m}$, $2961 \mathrm{~m}, 2874 \mathrm{~m}, 2807 \mathrm{~m}, 1559 \mathrm{~m}, 1497 \mathrm{~m}, 1453 \mathrm{~m}, 1203 \mathrm{~m}, 1058 \mathrm{~m}, 846 \mathrm{w}, 765 \mathrm{~m}, 703 \mathrm{~s}$. ${ }^{1} \mathrm{H}_{\mathrm{NMR}}\left(\mathrm{CDCl}_{3}\right)$ : ‘ 8.91 (s, 1H, $\left.\mathrm{HC}(2)\right) ; 7.55-7.50$ (m, 3H, $\mathrm{HC}(\operatorname{arom}$ )); 7.29-7.26 (m, 4H, HC(arom.)); 7.24-7.21 (m, 1H, HC(arom.)); 7.17-7.15 (m, 2H, HC(arom.)); 4.09-4.07 (m, 2H, $\mathrm{H}_{2} \mathrm{C}\left(\mathrm{alk}\right.$.)); $3.97\left(\mathrm{dd},{ }^{2} J_{\mathrm{H}, \mathrm{H}}=14.0,{ }^{3} J_{\mathrm{H}, \mathrm{H}}=5.4,1 \mathrm{H}, \mathrm{H}_{2} \mathrm{C}-\mathrm{N}\right) ; 3.93$ $\left(\mathrm{dd},{ }^{2} J_{\mathrm{H}, \mathrm{H}}=14.0,{ }^{3} J_{\mathrm{H}, \mathrm{H}}=6.6,1 \mathrm{H}, \mathrm{H}_{2} \mathrm{C}-\mathrm{N}\right) ; 3.52,3.43\left(\mathrm{AB}, J_{\mathrm{AB}}=13.2,2 \mathrm{H}, \mathrm{H}_{2} \mathrm{C}-\mathrm{Ph}\right)$; 3.00-2.96 (m, 1H, $\mathrm{H}_{2} \mathrm{C}\left(5^{\prime}\right)$ ); 2.93-2.88 (m, 1H, $\mathrm{H}_{2} \mathrm{C}\left(2^{\prime}\right)$ ); 2.43-2.37 (m, 1H, $\mathrm{H}_{2} \mathrm{C}\left(5^{\prime}\right)$ ); 2.16 (s, 3H, H $\left.{ }_{3} \mathrm{C}-\mathrm{C}(4)\right)$; 1.86-1.76 (m, 1H, $\mathrm{H}_{2} \mathrm{C}\left(3^{\prime}\right.$ ) , 2H, $\mathrm{H}_{2} \mathrm{C}($ alk.)); 1.74-1.66 (m, 1H, $\left.\mathrm{H}_{2} \mathrm{C}\left(4^{\prime}\right)\right)$; 1.60-1.53 (m, 1H, $\mathrm{H}_{2} \mathrm{C}\left(4^{\prime}\right)$ ); 1.44-1.38 (m, 2H, $\mathrm{H}_{2} \mathrm{C}($ alk.)); 1.36-1.31 (m, $\left.1 \mathrm{H}, \mathrm{H}_{2} \mathrm{C}\left(3^{\prime}\right)\right) ; 0.97\left(\mathrm{t}, J_{\mathrm{H}, \mathrm{H}}=7.50 \mathrm{~Hz}, \mathrm{H}_{3} \mathrm{C}(\right.$ alk. $\left.)\right) .{ }^{13} \mathrm{C} \mathrm{NMR}\left(\mathrm{CDCl}_{3}\right)$ : 139.3 (1 C(arom.)); 136.2 (HC(2)), 131.7, 130.9, 130.7, 129.7, 128.9, 128.5, 127.5, 127.3, 125.6 (10 HC(arom.), 1 C(arom.), 2 C(imid.)); 62.6 (HC(2')); $59.9\left(\mathrm{CH}_{2}-\mathrm{Ph}\right) ; 54.4\left(\mathrm{H}_{2} \mathrm{C}\left(5^{\prime}\right)\right)$; $50.9\left(\mathrm{CH}_{2}-\mathrm{N}\right)$; 47.6, 31.8 (2 C(alk.)); $28.4\left(\mathrm{C}\left(3^{\prime}\right)\right) ; 23.7$ (C(4')); 19.8 (C(alk.)); 13.6 $\left(\mathrm{H}_{3} \mathrm{C}(\right.$ alk. $\left.)\right) ; 8.9\left(\mathrm{H}_{3} \mathrm{C}-\mathrm{C}(4)\right)$. $[ \pm]_{\mathrm{D}}{ }^{25}=-10\left(c=1.0, \mathrm{CH}_{2} \mathrm{Cl}_{2}\right)$. 


\subsubsection{1- $\{[(2 S)-N$-Benzylpyrrolidin-2-yl]methyl $\}-4-m e t h y l-3-o c t y l-5-p h e n y l-1 H$ - imidazolium tetrafluoroborate 11d}

Yield: $1.9 \mathrm{~g}(72 \%)$. Pale yellow oil $\left(\mathrm{Al}_{2} \mathrm{O}_{3}, \mathrm{CHCl}_{3}\right)$. IR (film): v 3627w, 3557w, $3151 \mathrm{~m}, 3062 \mathrm{~m}, 2927 \mathrm{~m}, 2857 \mathrm{~m}, 2804 \mathrm{~m}, 1966 \mathrm{~m}, 1900 \mathrm{~m}, 1822 \mathrm{~m}, 1559 \mathrm{~m}, 1496 \mathrm{~m}$, 1459m, 1208m, 1058m, 926m, 843m, 763s, 703m. ${ }^{1} \mathrm{H}$ NMR $\left(\mathrm{CDCl}_{3}\right){ }^{\prime} 9.00$ (s, $1 \mathrm{H}$, $\mathrm{HC}(2))$; 7.55-7.50 (m, 3H, HC(arom.)); 7.29-7.26 (m, 4H, HC(arom.)); 7.24-7.21 (m, $1 \mathrm{H}, \mathrm{HC}($ arom. $)) ; 7.18-7.16(\mathrm{~m}, 2 \mathrm{H}, \mathrm{HC}(\operatorname{arom}).) ; 3.99\left(\mathrm{dd},{ }^{2} J_{\mathrm{H}, \mathrm{H}}=14.0,{ }^{3} J_{\mathrm{H}, \mathrm{H}}=5.4,1 \mathrm{H}\right.$, $\left.\mathrm{H}_{2} \mathrm{C}-\mathrm{N}\right) ; 3.94\left(\mathrm{dd},{ }^{2} J_{\mathrm{H}, \mathrm{H}}=14.0,{ }^{3} J_{\mathrm{H}, \mathrm{H}}=6.6,1 \mathrm{H}, \mathrm{H}_{2} \mathrm{C}-\mathrm{N}\right) ; 3.54,3.43\left(\mathrm{AB}, J_{A B}=13.2\right.$, 2H, $\left.\mathrm{H}_{2} \mathrm{C}-\mathrm{Ph}\right) ; 3.00-2.96$ (m, 1H, $\mathrm{H}_{2} \mathrm{C}\left(5^{\prime}\right)$ ); 2.94-2.90 (m, 1H, $\mathrm{HC}\left(2^{\prime}\right)$ ); 2.42-2.37 (m, $1 \mathrm{H}, \mathrm{H}_{2} \mathrm{C}\left(5^{\prime}\right)$ ); 2.16 (s, 3H, $\left.\mathrm{H}_{3} \mathrm{C}-\mathrm{C}(5)\right) ; 1.87-1.77$ (m, 2H, $\mathrm{H}_{2} \mathrm{C}\left(\right.$ alk.), 1H, $\mathrm{H}_{2} \mathrm{C}\left(4^{\prime}\right)$ ); 1.73-1.67 (m, 1H, $\left.\mathrm{H}_{2} \mathrm{C}\left(3^{\prime}\right)\right)$; 1.60-1.52 (m, 1H, $\left.\mathrm{H}_{2} \mathrm{C}\left(3^{\prime}\right)\right)$; 1.39-1.21 (m, 10H, $\mathrm{H}_{2} \mathrm{C}\left(\right.$ alk.) $\left., 1 \mathrm{H}, \mathrm{H}_{2} \mathrm{C}\left(4^{\prime}\right)\right) ; 0.87$ (t, $J_{\mathrm{H}, \mathrm{H}}=7.2, \mathrm{H}_{3} \mathrm{C}($ alk. $\left.)\right) .{ }^{13} \mathrm{C} \mathrm{NMR}\left(\mathrm{CDCl}_{3}\right){ }^{\prime} 139.3$ (1 $\mathrm{C}($ arom.); 136.2 (HC(2)); 131.7, 130.8, 130.7, 129.6, 128.9, 128.5, 127.5, 127.2, 125.5 (10 HC(arom.), 2 C(arom.) 2 C(imid.)); 62.6 (C(2')); $59.9\left(\mathrm{CH}_{2}-\mathrm{Ph}\right) ; 54.4\left(\mathrm{H}_{2} \mathrm{C}\left(5^{\prime}\right)\right)$; $50.8\left(\mathrm{CH}_{2}-\mathrm{N}\right)$; 47.8, 31.9, 29.9, 29.2 (4 C(alk.)); 29.2 (C(3')); 28.3, 26.6 (2 C(alk.)); $23.7\left(\mathrm{C}\left(4^{\prime}\right)\right) ; 22.8(\mathrm{C}($ alk. $)) ; 14.2\left(\mathrm{H}_{3} \mathrm{C}(\right.$ alk. $\left.)\right) ; 8.9\left(\mathrm{H}_{3} \mathrm{C}-\mathrm{C}(5)\right) .[ \pm]_{\mathrm{D}}{ }^{25}=-27(c=1.0$, $\mathrm{CH}_{2} \mathrm{Cl}_{2}$ ).

\subsubsection{Synthesis of 3-alkoxyimidazolium tetrafluoroborates 13}

A solution of an imidazole $N$-oxide 5 (5 mmol) and the corresponding alkyl bromide (5 $\mathrm{mmol})$ in $\mathrm{CHCl}_{3}(10 \mathrm{ml})$ was magnetically stirred at $\mathrm{rt}$ for $72 \mathrm{~h}$. Then, the solvent was removed under reduced pressure and the crude product was washed with $\mathrm{Et}_{2} \mathrm{O}(3 \times 5 \mathrm{ml})$, and dried under high vacuum for $4 \mathrm{~h}$. The imidazolium bromide obtained was magnetically stirred with an equimolar amount of solid $\mathrm{NaBF}_{4}$ in acetone $(5 \mathrm{ml})$ at $\mathrm{rt}$ overnight. The resulting suspension was filtered through neutral alumina $\left(\mathrm{Et}_{2} \mathrm{O}\right)$ and the solvent evaporated under reduced pressure.

\subsubsection{1. $1-\{[(2 S)-N$-Benzylpyrrolidin-2-yl $]$ methyl $\}-3-b u t o x y-4,5-d i m e t h y l-1 H$ - imidazolium tetrafluoroborate 13a}

Yield: $1.8 \mathrm{~g}(78 \%)$. Pale yellow oil $\left(\mathrm{Al}_{2} \mathrm{O}_{3}, \mathrm{CHCl}_{3}\right)$. IR (film): $v 3585 \mathrm{w}, 3380 \mathrm{~m}$, $3140 \mathrm{~m}, 2961 \mathrm{~m}, 2874 \mathrm{~m}, 2807 \mathrm{~m}, 1684 \mathrm{~m}, 1545 \mathrm{~m}, 1496 \mathrm{~m}, 1454 \mathrm{~m}, 1259 \mathrm{w}, 1187 \mathrm{w}$, 1058m, 936m, 848m, 820m, 735s, 703s, 613w. ${ }^{1} \mathrm{H}$ NMR $\left(\mathrm{CDCl}_{3}\right)$ :' $9.28(\mathrm{~s}, 1 \mathrm{H}$, $\mathrm{HC}(2))$; 7.31-7.14 (m, 5H, HC(arom.)); 4.38-4.29 (m, 2H, $\mathrm{H}_{2} \mathrm{C}($ alk. $\left.)\right) ; 4.09$ (dd, ${ }^{2} J_{\mathrm{H}, \mathrm{H}}=$ 
$\left.14.4,{ }^{3} J_{\mathrm{H}, \mathrm{H}}=4.8,1 \mathrm{H}, \mathrm{H}_{2} \mathrm{C}-\mathrm{N}\right) ; 3.96\left(\mathrm{dd},{ }^{2} J_{\mathrm{H}, \mathrm{H}}=14.4,{ }^{3} J_{\mathrm{H}, \mathrm{H}}=7.8,1 \mathrm{H}, \mathrm{H}_{2} \mathrm{C}-\mathrm{N}\right) ; 3.59$, $3.56\left(\mathrm{AB}, J_{A B}=13.2,1 \mathrm{H}, \mathrm{H}_{2} \mathrm{C}-\mathrm{Ph}\right) ; 3.19-3.11\left(\mathrm{~m}, 1 \mathrm{H}, \mathrm{H}_{2} \mathrm{C}\left(5^{\prime}\right), 1 \mathrm{H}, \mathrm{HC}\left(2^{\prime}\right)\right) ; 2.57-$ 2.52 (m, 1H, $\mathrm{H}_{2} \mathrm{C}\left(5^{\prime}\right)$ ); 2.15 (s, 3H, $\left.\mathrm{H}_{3} \mathrm{C}-\mathrm{C}(4)\right)$ ) 2.08 (s, 3H, $\mathrm{H}_{3} \mathrm{C}-\mathrm{C}(5)$ ); 2.06-2.00 (m, $1 \mathrm{H}, \mathrm{H}_{2} \mathrm{C}\left(3^{\prime}\right)$ ); 1.86-1.79 (m, 1H, $\mathrm{H}_{2} \mathrm{C}\left(4^{\prime}\right)$ ); 1.79-1.71 (m, 2H, $\mathrm{H}_{2} \mathrm{C}\left(\right.$ alk.), 1H, $\mathrm{H}_{2} \mathrm{C}\left(4^{\prime}\right)$ ); 1.56-1.44 (m, 2H, $\mathrm{H}_{2} \mathrm{C}\left(\right.$ alk.); $1 \mathrm{H}, \mathrm{H}_{2} \mathrm{C}\left(3^{\prime}\right)$ ); 0.97 (t, $J_{\mathrm{H}, \mathrm{H}}=7.2, \mathrm{H}_{3} \mathrm{C}($ alk. $)$ ). ${ }^{13} \mathrm{C} \mathrm{NMR}$ $\left(\mathrm{CDCl}_{3}\right)$ : ' 139.2 (1 C(arom.)); 131.7 (HC(2)); 129.0, 128.5, 127.3, 124.8, 123.5 (5 CH(arom.), 2 C(imid.)); 82.9 (C(alk.)); 62.5 (C(2’)); $60.6\left(\mathrm{CH}_{2}-\mathrm{Ph}\right) ; 55.0$ (C(5')); 50.9 $\left(\mathrm{CH}_{2}-\mathrm{N}\right) ; 29.9$ (C(alk.)); 28.3 (C(3')); 24.0 (C(4')); 19.0 (C(alk.)); 13.9 ( $\mathrm{H}_{3} \mathrm{C}($ alk.)); $8.7\left(\mathrm{H}_{3} \mathrm{C}-\mathrm{C}(5)\right) ; 7.2\left(\mathrm{H}_{3} \mathrm{C}-\mathrm{C}(4)\right)$. [ $]_{\mathrm{D}}{ }^{25}=-33\left(c=1.0, \mathrm{CH}_{2} \mathrm{Cl}_{2}\right)$.

\subsubsection{2. $1-\{[(2 S)-N$-Benzylpyrrolidin-2-yl]methyl $\}-4,5$-dimethyl-3-octyloxy-1H- imidazolium tetrafluoroborate 13b}

Yield: $1.7 \mathrm{~g}(69 \%)$. Pale yellow oil $\left(\mathrm{Al}_{2} \mathrm{O}_{3}, \mathrm{CHCl}_{3}\right)$. IR (film): v 3584w, 3566w, $3384 \mathrm{~m}, 3139 \mathrm{~m}, 3061 \mathrm{~m}, 2928 \mathrm{~m}, 2857 \mathrm{~m}, 2807 \mathrm{~m}, 1634 \mathrm{~m}, 1546 \mathrm{~m}, 1496 \mathrm{w}, 1454 \mathrm{w}$, 1260w, 1059m, 945m, 807w, 736m, 703s, 648w. ${ }^{1} \mathrm{H}$ NMR $\left(\mathrm{CDCl}_{3}\right)$ : ' $8.91(\mathrm{~s}, 1 \mathrm{H}$, $\mathrm{HC}(2))$; 7.27-7.19 (m, 3H, HC(arom.)); 7.17-7.13 (m, 2H, HC(arom.)); 4.36-4.27 (m, $2 \mathrm{H}, \mathrm{H}_{2} \mathrm{C}\left(\right.$ alk. )); $4.05\left(\mathrm{dd},{ }^{2} J_{\mathrm{H}, \mathrm{H}}=14.4,{ }^{3} J_{\mathrm{H}, \mathrm{H}}=4.8,1 \mathrm{H}, \mathrm{H}_{2} \mathrm{C}-\mathrm{N}\right) ; 3.93\left(\mathrm{dd},{ }^{2} J_{\mathrm{H}, \mathrm{H}}=14.4\right.$, $\left.{ }^{3} J_{\mathrm{H}, \mathrm{H}}=7.8,1 \mathrm{H}, \mathrm{H}_{2} \mathrm{C}-\mathrm{N}\right) ; 3.57,3.55\left(\mathrm{AB}, J_{A B}=13.2,2 \mathrm{H}, \mathrm{H}_{2} \mathrm{C}-\mathrm{Ph}\right) ; 3.16-3.10(\mathrm{~m}, 1 \mathrm{H}$, $\mathrm{H}_{2} \mathrm{C}\left(5^{\prime}\right), 1 \mathrm{H}, \mathrm{HC}\left(2^{\prime}\right)$ ); 2.57-2.52 (m, 1H, $\mathrm{H}_{2} \mathrm{C}\left(5^{\prime}\right)$ ); 2.15 (s, 3H, $\left.\mathrm{H}_{3} \mathrm{C}-\mathrm{C}(4)\right) ; 2.08$ (s, $\left.3 \mathrm{H}, \mathrm{H}_{3} \mathrm{C}-\mathrm{C}(5)\right)$; 2.07-2.01 (m, 1H, $\mathrm{H}_{2} \mathrm{C}\left(3^{\prime}\right)$ ); 1.86-1.80 (m, 1H, $\mathrm{H}_{2} \mathrm{C}\left(4^{\prime}\right)$ ); 1.78-1.71 (m, 2H, $\mathrm{H}_{2} \mathrm{C}\left(\right.$ alk.); 1H, $\left.\mathrm{H}_{2} \mathrm{C}\left(4^{\prime}\right)\right)$; 1.54-1.49 (m, 1H, $\left.\mathrm{H}_{2} \mathrm{C}\left(3^{\prime}\right)\right)$; 1.56-1.40 (m, 2H, $\mathrm{H}_{2} \mathrm{C}\left(\right.$ alk. )); $1.35-1.24$ (m, 8H, $\mathrm{H}_{2} \mathrm{C}($ alk. $\left.)\right) ; 0.89$ (t, $J_{\mathrm{H}, \mathrm{H}}=7.2,3 \mathrm{H}, \mathrm{H}_{3} \mathrm{C}\left(\right.$ alk.)). ${ }^{13} \mathrm{C} \mathrm{NMR}$ $\left(\mathrm{CDCl}_{3}\right)$ : ' 139.4 (1 C(arom.)); 131.3 (HC(2)); 129.0, 128.5, 127.3, 124.8, 123.5 (5 C(arom.), 2 C(imid.)); 62.4 (HC(2')); $60.6\left(\mathrm{CH}_{2}-\mathrm{Ph}\right) ; 54.9\left(\mathrm{C}\left(5^{\prime}\right)\right) ; 50.9\left(\mathrm{CH}_{2}-\mathrm{N}\right)$; 29.4, 28.3 (2 C(alk.)); 28.0 (C(3’)); 25.7 (C(alk.)); 24.0 (C(4')); 22.8 (C(alk.)); 14.3 $\left(\mathrm{H}_{3} \mathrm{C}(\right.$ alk. $\left.)\right) ; 8.7\left(\mathrm{H}_{3} \mathrm{C}-\mathrm{C}(5)\right), 7.3\left(\mathrm{H}_{3} \mathrm{C}-\mathrm{C}(4)\right)$. $[ \pm]_{\mathrm{D}}{ }^{25}=-26\left(c=1.0, \mathrm{CH}_{2} \mathrm{Cl}_{2}\right)$.

\subsubsection{3. $1-\{[(2 S)-N$-Benzylpyrrolidin-2-yl]methyl $\}-3-$ butoxy-4-methyl-5-phenyl-1H- imidazolium tetrafluoroborate $13 \mathrm{c}$}

Yield: $1.6 \mathrm{~g}(70 \%)$. Pale yellow oil $\left(\mathrm{Al}_{2} \mathrm{O}_{3}, \mathrm{CHCl}_{3}\right)$. IR (film): v 3584w, 3366m, 3135m, 3061m, 2961m, 2874m, 2806m, 1679m, 1543m, 1496m, 1452m, 1380m, 1283w, 1058m, 934m, 822w, 756m, 736m, 702s. ${ }^{1} \mathrm{H}$ NMR $\left(\mathrm{CDCl}_{3}\right)$ : 9.08 (s, 1H, $\mathrm{HC}(2))$; 7.53-7.48 (m, 3H, HC(arom.)); 7.28-7.13 (m, 7H, HC(arom.)); 4.52-4.43 (m, $2 \mathrm{H}, \mathrm{H}_{2} \mathrm{C}($ alk. $\left.)\right) ; 4.04\left(\mathrm{dd},{ }^{2} J_{\mathrm{H}, \mathrm{H}}=14.4,{ }^{3} J_{\mathrm{H}, \mathrm{H}}=4.8,1 \mathrm{H}, \mathrm{H}_{2} \mathrm{C}-\mathrm{N}\right) ; 3.95\left(\mathrm{dd},{ }^{2} J_{\mathrm{H}, \mathrm{H}}=14.4\right.$, 
$\left.{ }^{3} J_{\mathrm{H}, \mathrm{H}}=7.8,1 \mathrm{H}, \mathrm{H}_{2} \mathrm{C}-\mathrm{N}\right) ; 3.57,3.45\left(\mathrm{AB}, J_{A B}=12.6,2 \mathrm{H}, \mathrm{H}_{2} \mathrm{C}-\mathrm{Ph}\right) ; 3.06-3.02(\mathrm{~m}, 1 \mathrm{H}$, $\left.\mathrm{H}_{2} \mathrm{C}\left(5^{\prime}\right)\right)$; 2.91-2.86 (m, 1H, $\left.\mathrm{HC}\left(2^{\prime}\right)\right)$; 2.52-2.47 (m, 1H, $\left.\mathrm{H}_{2} \mathrm{C}\left(5^{\prime}\right)\right)$; 2.16 (s, 3H, $\mathrm{H}_{3} \mathrm{C}-$ $\mathrm{C}(4))$; 1.86-1.79 (m, 2H, $\mathrm{H}_{2} \mathrm{C}\left(\right.$ alk.), 1H, $\mathrm{H}_{2} \mathrm{C}\left(3^{\prime}\right)$ ); 1.78-1.71 (m, 1H, $\mathrm{H}_{2} \mathrm{C}\left(4^{\prime}\right)$ ); 1.631.50 (m, 2H, $\mathrm{H}_{2} \mathrm{C}\left(\right.$ alk.); $\left.1 \mathrm{H}, \mathrm{H}_{2} \mathrm{C}\left(4^{\prime}\right)\right)$; $1.43-1.28$ (m, $1 \mathrm{H}, \mathrm{H}_{2} \mathrm{C}\left(3^{\prime}\right)$ ); 1.00 (t, $J_{\mathrm{H}, \mathrm{H}}=7.2$, $3 \mathrm{H}, \mathrm{H}_{3} \mathrm{C}($ alk. $\left.)\right) .{ }^{13} \mathrm{C} \mathrm{NMR}\left(\mathrm{CDCl}_{3}\right)$ : ' 139.3 (1 C(arom.)); 132.1 (HC(2)); 130.9, 130.8, 129.7, 129.1, 129.0, 128.5, 127.3, 124.9, 124.8 (10 HC(arom.); 1 C(arom.), 2 C(imid.)); 83.2 (C(alk.)); 62.3 (HC(2')); $60.1\left(\mathrm{CH}_{2}-\mathrm{Ph}\right) ; 54.4\left(\mathrm{C}\left(5^{\prime}\right)\right) ; 50.6\left(\mathrm{CH}_{2}-\mathrm{N}\right) ; 30.1$ $(\mathrm{C}($ alk. $)) ; 27.9\left(\mathrm{C}\left(4^{\prime}\right)\right) ; 23.8\left(\mathrm{C}\left(3^{\prime}\right)\right) ; 19.1,13.9(2 \mathrm{C}($ alk. $)) ; 7.5\left(\mathrm{H}_{3} \mathrm{C}-\mathrm{C}(4)\right) .[ \pm]_{\mathrm{D}}{ }^{25}=-$ $25\left(c=1.0, \mathrm{CH}_{2} \mathrm{Cl}_{2}\right)$.

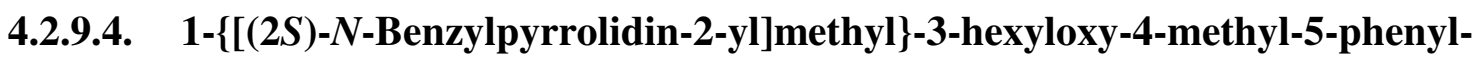
$1 H$-imidazolium tetrafluoroborane 13d

Yield: $1.9 \mathrm{~g}(76 \%)$. Pale yellow oil $\left(\mathrm{Al}_{2} \mathrm{O}_{3}, \mathrm{CHCl}_{3}\right)$. IR (film): $v 3584 \mathrm{w}, 3369 \mathrm{~m}$, $3135 \mathrm{~m}, 3061 \mathrm{~m}, 2930 \mathrm{~m}, 2872 \mathrm{~m}, 2807 \mathrm{~m}, 1683 \mathrm{~m}, 1542 \mathrm{~m}, 1496 \mathrm{~m}, 1453 \mathrm{~m}, 1379 \mathrm{~m}$, 1283w, 1058m, 933m, 913m, 765m, 735m, 702s. ${ }^{1} \mathrm{H}$ NMR $\left(\mathrm{CDCl}_{3}\right){ }^{\prime} 9.10$ (s, $1 \mathrm{H}$, $\mathrm{HC}(2))$; 7.55-7.48 (m, 3H, HC(arom.)); 7.29-7.14 (m, 7H, HC(arom.)); 4.51-4.42 (m, $2 \mathrm{H}, \mathrm{H}_{2} \mathrm{C}$ (alk.)); $4.04\left(\mathrm{dd},{ }^{2} J_{\mathrm{H}, \mathrm{H}}=14.0,{ }^{3} J_{\mathrm{H}, \mathrm{H}}=4.8,1 \mathrm{H}, \mathrm{H}_{2} \mathrm{C}-\mathrm{N}\right) ; 3.95\left(\mathrm{dd},{ }^{2} J_{\mathrm{H}, \mathrm{H}}=14.0\right.$, $\left.{ }^{3} J_{\mathrm{H}, \mathrm{H}}=7.8,1 \mathrm{H}, \mathrm{H}_{2} \mathrm{C}-\mathrm{N}\right) ; 3.57,3.45\left(\mathrm{AB}, J_{A B}=13.2,2 \mathrm{H}, \mathrm{H}_{2} \mathrm{C}-\mathrm{Ph}\right) ; 3.06-3.02(\mathrm{~m}, 1 \mathrm{H}$, $\left.\mathrm{H}_{2} \mathrm{C}\left(5^{\prime}\right)\right)$; 2.92-2.87 (m, 1H, $\left.\mathrm{HC}\left(2^{\prime}\right)\right)$; 2.53-2.47 (m, 1H, $\left.\mathrm{H}_{2} \mathrm{C}\left(5^{\prime}\right)\right)$; 2.16 (s, 3H, $\mathrm{H}_{3} \mathrm{C}-$ $\mathrm{C}(4))$; 1.87-1.80 (m, 2H, $\mathrm{H}_{2} \mathrm{C}\left(\right.$ alk.); $1 \mathrm{H}, \mathrm{H}_{2} \mathrm{C}\left(3^{\prime}\right)$ ); 1.78-1.71 (m, 1H, $\mathrm{H}_{2} \mathrm{C}\left(4^{\prime}\right)$ ); $1.64-$ 1.57 (m, 1H, $\mathrm{H}_{2} \mathrm{C}\left(4^{\prime}\right)$ ); 1.51-1.46 (m, 2H, $\mathrm{H}_{2} \mathrm{C}\left(\right.$ alk.)); 1.37-1.28 (m, 4H, $\mathrm{H}_{2} \mathrm{C}($ alk.), $1 \mathrm{H}$, $\left.\mathrm{H}_{2} \mathrm{C}\left(3^{\prime}\right)\right) ; 0.92\left(\mathrm{t}, J_{\mathrm{H}, \mathrm{H}}=6.9,3 \mathrm{H}, \mathrm{H}_{3} \mathrm{C}(\right.$ alk. $\left.)\right) .{ }^{13} \mathrm{C} \mathrm{NMR}\left(\mathrm{CDCl}_{3}\right)$ : ' 139.3 (1 C(arom.)); 132.1 (HC(2)); 131.0, 130.9, 129.6, 129.1, 129.0, 128.5, 127.3, 124.9, 124.8 (10 HC(arom.); 1 C(arom.), 2 C(imid.)); 83.5 (C(alk.)); 62.3 (HC(2')); $60.1\left(\mathrm{CH}_{2}-\mathrm{Ph}\right) ; 54.4$ $\left(\mathrm{C}\left(5^{\prime}\right)\right)$; $50.6\left(\mathrm{CH}_{2}-\mathrm{N}\right)$; 31.6, 28.1 (2 C(alk.)); 27.9 (C(3’)); 25.4 (C(alk.)); 23.8 (C(4')); 22.7 (C(alk.)); $14.2\left(\mathrm{H}_{3} \mathrm{C}(\right.$ alk. $\left.)\right) ; 7.6\left(\mathrm{H}_{3} \mathrm{C}-\mathrm{C}(4)\right)$. $[ \pm]_{\mathrm{D}}{ }^{25}=-17\left(c=1.0, \mathrm{CH}_{2} \mathrm{Cl}_{2}\right)$.

\subsubsection{1 - $\{[(2 S)-N$-Benzylpyrrolidin-2-yl $]$ methyl $\}-3-b u t o x y-4,5-d i p h e n y l-1 H$ -} imidazolium tetrafluoroborate $13 \mathrm{e}$

Yield: $1.5 \mathrm{~g}(53 \%)$. Pale yellow oil $\left(\mathrm{Al}_{2} \mathrm{O}_{3}, \mathrm{CHCl}_{3}\right)$. IR (film): $v 3648 \mathrm{w}, 3584 \mathrm{w}$, 3369w, 3135m, 3061m, 2961m, 2874m, 2808m, 1683m, 1494m, 1447m, 1271m, 1179m, 1058m, 934m, 819m, 763m, 735m, 700m, 607w. ${ }^{1} \mathrm{H}$ NMR $\left(\mathrm{CDCl}_{3}\right)$ : $^{\prime} 9.24$ (s, $1 \mathrm{H}, \mathrm{HC}(2)) ; 7.50-7.20$ (m, 15H, HC(arom.)); 4.26-4.19 (m, 1H, $\mathrm{H}_{2} \mathrm{C}-\mathrm{N}, 2 \mathrm{H}$, $\mathrm{H}_{2} \mathrm{C}$ (alk.)); $4.10\left(\mathrm{dd},{ }^{2} J_{\mathrm{H}, \mathrm{H}}=13.8,{ }^{3} J_{\mathrm{H}, \mathrm{H}}=7.2,1 \mathrm{H}, \mathrm{H}_{2} \mathrm{C}-\mathrm{N}\right) ; 3.65,3.49\left(\mathrm{AB}, J_{A B}=13.2\right.$, 
2H, $\left.\mathrm{H}_{2} \mathrm{C}-\mathrm{Ph}\right) ; 3.06-3.01$ (m, 1H, $\left.\mathrm{H}_{2} \mathrm{C}\left(5^{\prime}\right)\right)$; 2.98-2.92 (m, 1H, $\left.\mathrm{HC}\left(2^{\prime}\right)\right)$; 2.53-2.47 (m, $\left.1 \mathrm{H}, \mathrm{H}_{2} \mathrm{C}\left(5^{\prime}\right)\right)$ ) 1.89-1.82 (m, 1H, $\mathrm{H}_{2} \mathrm{C}\left(3^{\prime}\right)$ ); 1.79-1.73 (m, 1H, $\mathrm{H}_{2} \mathrm{C}\left(4^{\prime}\right)$ ); 1.64-1.56 (m, $2 \mathrm{H}, \mathrm{H}_{2} \mathrm{C}\left(\right.$ alk.); 1H, $\left.\mathrm{H}_{2} \mathrm{C}\left(4^{\prime}\right)\right)$; 1.36-1.32 (m, 1H, $\left.\mathrm{H}_{2} \mathrm{C}\left(3^{\prime}\right)\right)$; 1.31-1.24 (m, 2H, $\mathrm{H}_{2} \mathrm{C}($ alk. $\left.)\right) ; 0.79$ (t, $J_{\mathrm{H}, \mathrm{H}}=7.5,3 \mathrm{H}, \mathrm{H}_{3} \mathrm{C}($ alk. $\left.)\right) .{ }^{13} \mathrm{C} \mathrm{NMR}\left(\mathrm{CDCl}_{3}\right)$ : ' 139.3 (1 C(arom.)); 133.1 (HC(2)); 131.3, 130.9, 130.4, 129.8, 129.73, 129.68, 129.2, 129.1, 128.7, 127.6, 126.8, 124.6, 123.4 (15 HC(arom.), 2 C(arom.) 2 C(imid.)); 83.4 (C(alk.)); 62.3 (C(2’)); $59.8\left(\mathrm{CH}_{2}-\mathrm{Ph}\right) ; 54.1\left(\mathrm{C}\left(5^{\prime}\right)\right) ; 50.4\left(\mathrm{CH}_{2}-\mathrm{N}\right) ; 29.8$ (C(alk.)); $23.6\left(\mathrm{C}\left(3^{\prime}\right)\right) ; 18.8$ $\left(\mathrm{C}\left(4^{\prime}\right)\right) ; 13.7\left(\mathrm{H}_{3} \mathrm{C}(\mathrm{alk}).\right) .[ \pm]_{\mathrm{D}}^{25}=-16\left(c=1.0, \mathrm{CH}_{2} \mathrm{Cl}_{2}\right)$.

\section{Acknowledgments}

A. W. thanks National Science Center (Cracow) for financial support (Grant Preludium \# UMO-2012/07/N/ST5). The authors thank PD Dr. L. Bigler, University of Zürich, for ESI-HRMS. 


\section{References}

[1] Part of the Diploma thesis, University of Aódz, 2011, and of the planned PhD Thesis of A. W., University of Aódz.

[2] (a) Katrizky, A. R.; Ramsden, C. A.; Scriven, E. F. V.; Taylor, R. J. K. Comprehensive Heterocyclic Chemistry III; Five-membered Rings with Two Heteroatoms, Each with Their Fused Carbocylic Derivatives, Vol. 4; Joule, J., Ed.; Elsevier: Oxford 2008; (b) Joule, J. Mills, K. Heterocyclic Chemistry, V Edition, J. Wiley \& Sons, Chichester 2010; (c) Bhatnagar A.; Sharma, P. K.; Kumar, N. Int. J. Pharm. Tech. Res. 2011, 3, 268-282.

[3] Babizhayev, M. A. Life Science 2006, 78, 2343-2357.

[4] Narasimhan, B.; Dharma, D.; Kumar, P. Med. Chem. Res. 2011, 20, 1119-1140.

[5] (a) Nikitina, G. V.; Pevzner, M. S. Chem. Heterocycl. Comp. 1993, 127-151; (b) Alcázar, J.; Begtrup, M.; de la Hoz, A,. J. Chem. Soc., Perkin Trans 1 1995, 24672470; (c) Laufer, S.; Wagner, G.; Kotschenreuther, D. Angev. Chem. Int. Ed. 2002, 41, 2290-2293; (d) Aquirre, G.; Boiani, M.; Cerecetto, H.; Gerpe, A.; Gonzales, M.; Fernandez Sainz, Y.; Denicola, A.; Ochoa de Ocariz, C.; Nogal, J. J.; Montero, D.; Escario, J. A. Arch. Pharm. Pharm. Med. Chem. 2004, 337, 259270.

[6] Laus, G.; Schwärzler, A.; Bentivoglio, G.; Hummel,, M.; Kahlenberg, V.; Wurst, K.; Kristeva, E.; Schütz, J.; Kopacka, H.; Kreuz, C.; Bonn, G.; Anfriyko, Y.; Nauer, G.; Schottenberger, H. Z. Z. Naturforsch. 2008, 63B, 447-464.

[7] Lettau, H. Z. Chem. 1970, 10, 211-216.

[8] (a) Mucha, P.; MlostoD, G.; JasiDski, M.; Linden, A.; Heimgartner, H. Tetrahedron: Asymmetry 2008, 19, 1600-1607; (b) MlostoD, G.; Mucha, P.; Urbaniak, K.; Broda, K.; Heimgartner, H. Helv. Chim. Acta, 2008, 91, 232-238; (c) JasiDski, M.; MlostoD, G.; Linden, A.; Heimgartner, H. Helv. Chim. Acta 2008, 91, 1916-1933; (d) MlostoD, G.; RomaDski, J.; JasiDski, M.; Heimgartner, H. Tetrahedron: Asymmetry 2009, 20, 1073-1080.

[9] Kwiatkowski, P.; Mucha, P.; MlostoD, G.; Jurczak, J. Synlett 2009, 1157-1760.

[10] MlostoD, G.; Mucha, P.; Heimgartner, H. Lett. Org. Chem. 2012, 9, 89-91.

[11] (a) Morris, D. J.; Partridge, A. S.; Manville, C. V.; Racys, D. T.; Woodward, G.; Docherty, G.; Wills, M. Tetrahedron Lett. 2010, 51, 209-212; b) Cao, X.-Y.; 
Zheng, J.-C.; Li, Y.-X,; Shu, Z.-C.; Sun, X.-L.; Wanf, B.-Q.; Tang, Y. Tetrahedron 2010, 66, 9703-9707.

[12] MlostoD, G,; Pieczonka, M. A.; Wróblewska, A.; Linden, A.; Heimgartner. H. Heterocycles 2012, 86, 343-356.

[13] Rispeus, M. T.; Gelling, O. J.; de Vries, A. H. M.; Meetsma, A.; van Bolhuis, F.; Feringa, B. L. Tetrahedron 1996, 52, 3521-3546.

[14] Ghandi, M.; Salimi, F.; Ohyaei, A. Molecules 2006, 11, 556-563.

[15] Mloston, G.; JasiDski, M. Arkivoc 2011, (vi), 162-175.

[16] (a) MlostoD, G.; Obijalska, E.; Tafelska-Kaczmarek, A.; Zaidlewicz, M. J. Fluorine Chem. 2010, 131, 1289-1296; (b) for a review, see: Vassiliou, S. Current Org. Chem. 2011, 15, 2469-2480.

[17] MlostoD, G.; Celeda, M.; Prakash, G. K. S.; Olah, G. A.; Heimgartner, H. Helv. Chim. Acta 2000, 83, 728-738.

[18] MlostoD, G.; JasiDski, M.; Heimgartner, H. Eur. J. Org. Chem. 2011, 2542-2547.

[19] MlostoD, G.; Mucha, P.; Tarka, R.; Urbaniak, K.; Linden, A.; Heimgartner, H.; Pol. J. Chem. 2009, 83, 1105-1114.

[20] (a) MlostoD, G.; Gendek, T.; Heimgartner, H.; Helv. Chim. Acta 1998, 81, 15851595; (b) MlostoD, G.; JasiDski, M.; Rygielska, D.; Heimgartner, H. Heterocycles 2011, 83, 765-776.

[21] (a) Zhang, Z.; Schreiner, P. R. Chem. Soc. Rev. 2009, 38, 1187-1198; (b) Doyle, A. G.; Jacobsen, E. N. Chem. Rev. 2007, 107, 5713-5743.

[22] (a) Wasserscheid, P.; Welton, T. Ionic Liquids in Synthesis; Wiley-VCH; Weinheim, 2008; (b) Bica, K.; Gaertner, P. Eur. J. Org. Chem. 2008, 3235-3250; (c) Chen, X.; Li, X.; Hu. A.; Wang, F. Tetrahedron: Asymmetry 2008, 19, 1-14; (d) Headly, A. D.; Ni, B. Aldrichim. Acta 2007, 40, 107-117.

[23] Xu, D.; Luo, S.; Yue, H.; Wang, L.; Lin, Y.; Xu, Z. Synlett 2006, 2569-2572.

[24] Belokon, Y. N.; Tararov, V. I.; Maleev, V. I.; Savel'eva, T. F.; Ryzhov, M. G. Tetrahedron: Asymmetry 1998, 9, 4249-4252.

[25] Itsuno, S.; Ito, K.; Hirao, A.; Nakahama, S. J. Chem. Soc. Perkin Transl. 1 1984, 2887-2893.

[26] Belokon, Y. N.; Maleev, V. I.; Videnskaya, S. O.; Saporovskaya, M. B.; Tsyryapkin, V. A.; Belikov, V. M. Bull. Acad. Sci. USSR, Div. Chem. Sci. (Engl. Transl.) 1991, 40, 110-118. 